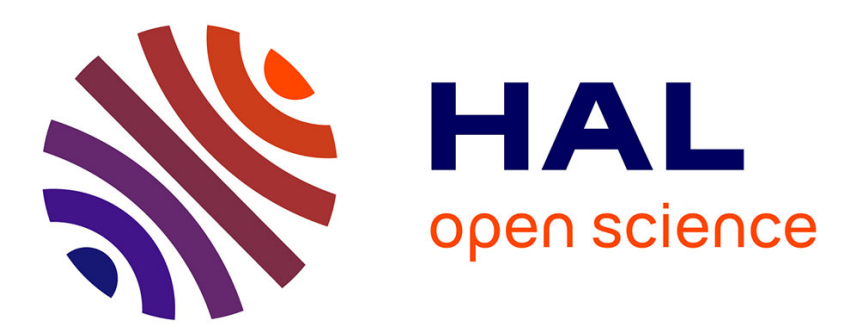

\title{
Geometric chaos indicators and computations of the spherical hypertube manifolds of the spatial circular restricted three-body problem
}

\author{
Massimiliano Guzzo, Elena Lega
}

\section{To cite this version:}

Massimiliano Guzzo, Elena Lega. Geometric chaos indicators and computations of the spherical hypertube manifolds of the spatial circular restricted three-body problem . 2017. hal-01343970v2

HAL Id: hal-01343970

https://hal.science/hal-01343970v2

Preprint submitted on 6 Apr 2017

HAL is a multi-disciplinary open access archive for the deposit and dissemination of scientific research documents, whether they are published or not. The documents may come from teaching and research institutions in France or abroad, or from public or private research centers.
L'archive ouverte pluridisciplinaire HAL, est destinée au dépôt et à la diffusion de documents scientifiques de niveau recherche, publiés ou non, émanant des établissements d'enseignement et de recherche français ou étrangers, des laboratoires publics ou privés. 


\title{
Geometric chaos indicators and computations of the spherical hypertube manifolds of the spatial circular restricted three-body problem
}

\author{
Massimiliano Guzzo \\ Università degli Studi di Padova \\ Dipartimento di Matematica "Tullio Levi-Civita" \\ Via Trieste, 63 - 35121 Padova, Italy \\ guzzo@math.unipd.it \\ Elena Lega \\ Laboratoire Lagrange, Université Côte d'Azur \\ Observatoire de la Côte d'Azur, CNRS \\ Blvd de l'Observatoire, CS 34229, 06304 Nice cedex 4, France \\ elena.lega@oca.eu
}

April 6, 2017

\begin{abstract}
The circular restricted three-body problem has five relative equilibria $L_{1}, L_{2}, \ldots, L_{5}$. The invariant stable-unstable manifolds of the center manifolds originating at the partially hyperbolic equilibria $L_{1}, L_{2}$ have been identified as the separatrices for the motions which transit between the regions of the phase-space which are internal or external with respect to the two massive bodies. While the stable and unstable manifolds of the planar problem have been extensively studied both theoretically and numerically, the spatial case has not been as deeply investigated. This paper is devoted to the global computation of these manifolds in the spatial case with a suitable finite time chaos indicator. The definition of the chaos indicator is not trivial, since the mandatory use of the regularizing Kustaanheimo-Stiefel variables introduces discontinuities in the individual Lyapunov indicators. From the study of such discontinuities, we define geometric chaos indicators which are smooth, globally defined, and whose ridges sharply approximate the stable and unstable manifolds of the center manifolds of $L_{1}, L_{2}$. We illustrate the method for the Sun-Jupiter mass ratio, and represent the topology of the asymptotic manifolds using sections and three-dimensional representations.
\end{abstract}




\section{Introduction}

The circular restricted three-body problem describes the motion of a massless body $P$ in the gravitation field of two massive bodies $P_{1}$ and $P_{2}$, called primary and secondary body respectively, which rotate uniformly around their common center of mass. In a rotating frame the problem has five equilibria, the so called Lagrangian points $L_{1}, \ldots, L_{5}$, which are the only known simple solutions of the equations of motion of $P$ :

$$
\left\{\begin{array}{l}
\ddot{x}=2 \dot{y}+x-(1-\mu) \frac{x+\mu}{r_{1}^{3}}-\mu \frac{x-1+\mu}{r_{2}^{3}} \\
\ddot{y}=-2 \dot{x}+y-(1-\mu) \frac{y}{r_{1}^{3}}-\mu \frac{\frac{y}{r_{2}^{3}}}{\ddot{z}}=-(1-\mu) \frac{z}{r_{1}^{3}}-\mu \frac{z}{r_{2}^{3}}
\end{array}\right.
$$

where the units of masses, lengths and time have been chosen so that the masses of $P_{1}$ and $P_{2}$ are $1-\mu$ and $\mu(\mu \leq 1 / 2)$ respectively, their coordinates are $(-\mu, 0,0)$ and $(1-\mu, 0,0)$ and their revolution period is $2 \pi$; we denoted by $r_{1}=\sqrt{(x+\mu)^{2}+y^{2}+z^{2}}$ and by $r_{2}=$ $\sqrt{(x-1+\mu)^{2}+y^{2}+z^{2}}$ the distances of $P$ from $P_{1}, P_{2}$. As it is well known, equations (1) have an integral of motion, the so-called Jacobi constant, defined by:

$$
\mathcal{C}(x, y, z, \dot{x}, \dot{y}, \dot{z})=x^{2}+y^{2}+2 \frac{1-\mu}{r_{1}}+2 \frac{\mu}{r_{2}}-\dot{x}^{2}-\dot{y}^{2}-\dot{z}^{2} .
$$

Fixed values $C$ of the Jacobi constant define level sets $\mathcal{M}_{C}$ in the phase-space, which project on the set:

$$
\Pi \mathcal{M}_{C}=\left\{(x, y, z) \in \mathbb{R}_{0}^{3}: x^{2}+y^{2}+2 \frac{1-\mu}{r_{1}}+2 \frac{\mu}{r_{2}} \geq C\right\}
$$

of the physical space:

$$
\mathbb{R}_{0}^{3}=\left\{(x, y, z) \in \mathbb{R}^{3}:(x, y, z) \neq(-\mu, 0,0),(1-\mu, 0,0)\right\} .
$$

The boundary $\mathcal{B}_{C}$ of $\Pi \mathcal{M}_{C}$ separates the so called realm of possible motions $\Pi \mathcal{M}_{C}$ from the realm of forbidden motions $\mathbb{R}_{0}^{3} \backslash \Pi \mathcal{M}_{C}$.

The Lagrangian equilibria $L_{1}, \ldots, L_{5}$ are critical points for the Jacobi constant; the values $C_{1}, C_{2}, \ldots, C_{5}$ of $\mathcal{C}$ at the Lagrangian equilibria $L_{1}, \ldots, L_{5}$ correspond to topological changes of the set $\mathcal{B}_{C}$. In particular, for $C>C_{2}$ the space $\mathbb{R}_{0}^{3}$ is disconnected by $\mathcal{B}_{C}$ into a region of motions which contains the massive bodied $P_{1}, P_{2}$ and an external region; for $C<C_{2}$ the realm of possible motions is connected; in particular, for values of $C$ slightly smaller than $C_{2}$, the connection between the internal and external regions is realized through a bottleneck of $\mathcal{B}_{C}$, at whose extremities we find the Lagrangian points $L_{1}$ and $L_{2}$. The transit of motions through this bottleneck is guided by the stable-unstable manifolds of the center manifolds $W_{1}^{c}, W_{2}^{c}$ originating at the equilibria $L_{1}, L_{2}$, which are partially hyperbolic, specifically they are saddle $\times$ center $\times$ center. The center manifold theorem (see, for example, [31]) grants the existence of two four-dimensional 
center manifolds $W_{i}^{c}, i=1,2$. Since the restriction of the Jacobi constant to each $W_{i}^{C}$ has a strict extremum at the equilibrium point $L_{i}$, from the general results of [31] we obtain that for suitably small values of $C-C_{i}$ the sets $W_{C, i}^{c}=W_{i}^{c} \cap \mathcal{M}_{C}$ are unique, are diffeomorphic to a three-sphere, are invariant with respect to the flow of the three-body problem for any time $t \in \mathbb{R}$. Their stable and unstable manifolds have the topology of hypertubes obtained from the product of a three-sphere with an half line; we will call them spherical hypertube manifolds. The spherical hypertube manifolds act as separatrices for the transit of motions through the bottlenecks of $\mathcal{B}_{C}$ connecting the region of internal and the region of external motions, see $[2,32,17]$ (for the planar three-body problem) and $[9,4]$ (for the spatial three-body problem). This fact is a consequence of the structure of the local stable-unstable manifolds of $W_{C, i}^{c}$ in a small neighbourhood $\mathcal{U}_{i}$ : motions with initial conditions in $\mathcal{U}_{i}$ approaching the center manifold from the right-hand side (left-hand side respectively) 'bounce back' if they are on one side of the separatrix, while they transit to the left-hand side (right-hand side respectively) if they are on the other side of the separatrix.

The structure of the global stable and unstable manifolds of $W_{C, i}^{c}$ is much more complicate than the structure of the local manifolds: the exponential compressions, expansions and rotations occurring near the center manifolds are alternated to circulations around both primaries. Global representations of these surfaces have been obtained for several sample values of $\mu$ and $C$ in the planar circular restricted three-body problem, see for example [17, 13, 24]. The computation of the stable-unstable manifolds in the planar case has several advantages with respect to the spatial case. First, in the planar case, the level set of the center manifolds obtained by fixing the value of the Jacobi constant in a suitable small left neighbourhood of $C_{i}$ is made of a periodic orbit, the horizontal Lyapunov orbit of $L_{1}$ or $L_{2}$. To compute their asymptotic manifolds one can use one of the several methods of computation of the stable and unstable manifolds of periodic orbits, for example the flow continuation of the local manifolds, the parametrization method, or the recent method based on chaos indicators (see [13] and the references therein). Moreover, the phase-space of the planar three-body problem is four dimensional, and by fixing the value of the Jacobi constant we obtain a three dimensional space. The stable and unstable manifolds of the horizontal Lyapunov orbits are therefore two-dimensional surfaces in a three-dimensional space; their global phase-space development has been graphically visualized in [24].

In this paper we provide a method, based on the computation of chaos indicators, to compute and represent the global structure of the stable-unstable manifolds of $W_{C, i}^{c}$ in the spatial circular restricted three-body problem. While the computation of the stable and unstable manifolds of equilibria and of periodic orbits has been considered in many papers, much less attention has been devoted to the computation of the stable and unstable manifolds of center manifolds of higher dimension. Our method applies independently on the dimension of the center manifold, provided that it is globally invariant with respect to the flow: first, we construct a neighbourhood $\mathcal{U}_{i}$ of the center manifold $W_{C, i}^{c}$ where the local stable and unstable manifolds are represented as Cartesian graphs and an hyperbolic Birkhoff normal form of some convenient order is defined; then, we localize the global stable manifold $W_{C, i}^{s}$ by exploiting at the same time the peculiar 
growth of the tangent vectors close to $W_{C, i}^{c}$, and the scattering from $\mathcal{U}_{i}$ of the motions with initial conditions outside the local stable manifold. Both properties are coded by a finite time chaos indicator, fast Lyapunov indicator or finite time Lyapunov indicator, whose ridges provide the stable manifold ${ }^{1} W_{C, i}^{s}$.

The definition of smooth chaos indicators for the spatial three-body problem is non trivial since equations (1) are singular for $(x, y, z)=(-\mu, 0,0)$ or $(1-\mu, 0,0)$. Smooth indicators will be defined by using the Kustaanheimo-Stiefel regularization which has been originally introduced to regularize the spatial problem (KS hereafter, $[18,19])$. The use of regularizing transformations, with their analytic and computational advantages (see, for example, $[1,35]$ ) appears to us mandatory to compute long pieces of the spherical hypertube manifolds of $L_{1}, L_{2}$, especially close to the secondary body. In this spirit, the Levi-Civita transformation (LC hereafter), which regularizes the equations of motion of the planar three-body problem [27], has been used in [13, $24]$ to define chaos indicators whose ridges approximate the stable and unstable manifolds of the horizontal Lyapunov orbits of $L_{1}, L_{2}$. Even if the KS transformation is the natural extension of the LC transformation, the regularization of the spatial problem is intrinsically more complicate than the planar one. On the one hand, we show that as a consequence of Sundman's proper time transformation [34] used in KS and LC transformation, for both regularizations the solutions of the variational equations written in the Cartesian phase-space are not conjugate to the solutions of the variational equations written in the $\mathrm{KS}$ or LC variables, so that the Lyapunov exponents (as well as other chaos indicators) defined with the regularized variational equations are different functions from the chaos indicators defined with the non-regularized variational equations. On the other hand, the redundancy of the Kustaanheimo-Stiefel variables introduces a symmetry, degenerate at the singularity, whose consequence is twofold: two additional Lyapunov exponents are introduced; the chaos indicators defined by the KS-regularized variational equations for orbits corresponding to the same initial conditions of the physical phase-space and the same initial tangent vector have discontinuities. A careful analysis of these discontinuities allows us to define 'geometric' chaos indicators, which are globally defined and smooth on the Cartesian phase-space, and to suitably select a family of branched indicators whose ridges approximate the stable and unstable manifolds of $W_{C, i}^{c}$, to conveniently use for the numerical applications.

Once the suitable chaos indicators are defined, we set up a method to represent the global development in the phase-space of the hypertube manifolds. The most important problem is related to the dimension of the hypertubes and of the invariant set $\mathcal{M}_{C}$ they belong to. Our first choice is to compute and represent sections of the hypertubes, which provide us the knowledge of how these manifolds intersect well chosen two-dimensional surfaces transverse to it. Because of the symmetry of the three-body problem, the first condition to define a good section is to set: $y=0$. In the planar problem the condition $y=0$ defines the two-dimensional surface in $\mathcal{M}_{C}$ :

$$
\Sigma_{P}=\{(x, y, z, \dot{x}, \dot{y}, \dot{z}): y=z=0, \dot{z}=0, \mathcal{C}(x, 0, \dot{x}, \dot{y}, 0)=C\} .
$$

\footnotetext{
${ }^{1}$ As usual, the unstable manifold $W_{C, i}^{u}$ is obtained by computing the stable manifold of the inverse flow.
} 
which we call the planar section. In the planar section there are peculiar initial conditions, characterized by $\dot{x}=0$, whose orbits satisfy a well known time reversal symmetry. In particular, if they belong to the stable manifold of $L_{1}$ or $L_{2}$, they are homoclinic. The vertical section:

$$
\Sigma_{V}=\{(x, y, z, \dot{x}, \dot{y}, \dot{z}): y=0, \dot{x}=\dot{z}=0, \mathcal{C}(x, 0, \dot{x}, \dot{y}, 0)=C\}
$$

intersects the planar section $\Sigma_{P}$ exactly on its line $\dot{x}=0$. The homoclinic planar orbits of $L_{i}$ on this axes are continued in $\Sigma_{V}$ to curves of initial conditions on homoclinic orbits of the center manifold $W_{C, i}^{c}$. In fact, motions $(x(t), y(t), z(t))$ with $(x(0), y(0), z(0), \dot{x}(0), \dot{y}(0), \dot{z}(0)) \in \Sigma_{V}$ satisfy the time-reversal symmetry (also $(x(-t),-y(-t), z(-t))$ is a solution of equations (1)). Moreover, from the mirror theorem [29], if an orbit hits the section in at least two points, then it is a periodic orbit and therefore cannot belong to a stable or unstable manifold. As a consequence, the vertical section is not redundant: all the points of $W^{s} \cap \Sigma$ are not connected by orbits.

We will compute several connected components of $W_{C, i}^{s} \cap \Sigma_{V}$ of both Lagrangian points $L_{1}, L_{2}$, in the realms of motions around the primary and secondary body respectively. To define precisely the realms of motions for $C<C_{2}$ we find useful to consider isolating blocks at $L_{1}, L_{2}$ $[3,28,4]$ of equations:

$$
\begin{aligned}
& R_{1}=\left\{(x, y, z, \dot{x}, \dot{y}, \dot{z}) \in \mathcal{M}_{C}: x \in\left[x_{1}, x_{2}\right]\right\} \\
& R_{2}=\left\{(x, y, z, \dot{x}, \dot{y}, \dot{z}) \in \mathcal{M}_{C}: x \in\left[x_{3}, x_{4}\right]\right\}
\end{aligned}
$$

where $x_{1}<x_{L_{1}}<x_{2}, x_{3}<x_{L_{2}}<x_{4}$, while we have:

$$
x-(1-\mu) \frac{x+\mu}{r_{1}^{3}}-\mu \frac{x-1+\mu}{r_{2}^{3}}<-2 \sqrt{x^{2}+y^{2}+2 \frac{(1-\mu)}{r_{1}}+2 \frac{\mu}{r_{2}}-C}
$$

for all $\left(x_{1}, y, z\right) \in \Pi \mathcal{M}_{C}$ and $\left(x_{3}, y, z\right) \in \Pi \mathcal{M}_{C}$, and:

$$
x-(1-\mu) \frac{x+\mu}{r_{1}^{3}}-\mu \frac{x-1+\mu}{r_{2}^{3}}>2 \sqrt{x^{2}+y^{2}+2 \frac{(1-\mu)}{r_{1}}+2 \frac{\mu}{r_{2}}-C}
$$

for all $\left(x_{2}, y, z\right) \in \Pi \mathcal{M}_{C}$ and $\left(x_{4}, y, z\right) \in \Pi \mathcal{M}_{C}$. The two conditions are found so that any orbit hitting the border of $R_{1}$ or $R_{2}$ from the left (resp. from the right) tangentially, i.e. with $\dot{x}=0$, bounces back because $\ddot{x}<0$ (resp. because $\ddot{x}>0$ ). The center manifolds $W_{C, i}^{c}$ are contained in the isolating blocks $R_{i}$ and, by taking $x_{1}, x_{3}$ as large as possible and $x_{2}, x_{4}$ as small as possible (within their numerical error determination), we define the realms $S, J, E$ of motions around the primary, around the secondary mass ${ }^{2}$ and external to the binary system $P_{1} P_{2}$ by (see figure 1 )

$$
S=\Pi\left\{(x, y, z, \dot{x}, \dot{y}, \dot{z}) \in \mathcal{M}_{C}: x<x_{1}\right\}
$$

\footnotetext{
${ }^{2}$ The realm of motions around $P_{1}$ and $P_{2}$ are denoted by $S, J$ respectively, since we conventionally identify $P_{1}$ with the Sun and $P_{2}$ with Jupiter.
} 


$$
\begin{aligned}
J & =\Pi\left\{(x, y, z, \dot{x}, \dot{y}, \dot{z}) \in \mathcal{M}_{C}: x \in\left(x_{2}, x_{3}\right)\right\} \\
E & =\Pi\left\{(x, y, z, \dot{x}, \dot{y}, \dot{z}) \in \mathcal{M}_{C}: x>x_{4}\right\}
\end{aligned}
$$

respectively.

The connected components of $W_{C, i}^{s} \cap \Sigma_{V}$ of both Lagrangian points $L_{1}, L_{2}$, will be denoted by $\Lambda_{j}^{i}$ if they are in the realm $J$ of motions around the secondary mass; $i=1$ or $i=2$ depending on the Lagrangian point $L_{1}, L_{2} ; j=1,2 \ldots$ is a label for the connected component; the components will be denoted by $M_{j}^{1}$ if they are in the realm $S$ of motions around the primary mass defined by $x \leq x_{1}$.

Since we find that the $\Lambda_{j}^{i}$ are closed, and since the spherical hypertube manifolds are separatrices for the transit from/to the realm of motions $S, J$ and $E$, all the initial conditions of $\Sigma_{V}$ which are in the interior of $\Lambda_{j}^{1}$ transit from $J$ to $S$ in the same number of revolutions $(j-1) / 2$ around the secondary mass $P_{2}$; similarly we find that the initial conditions of $\Sigma_{V}$ which are in the interior of $\Lambda_{j}^{2}$ transit from $J$ to $E$ in the same number of revolutions $(j-1) / 2$ around the secondary mass $P_{2}$. The transits of the orbits with initial conditions in $\Sigma_{V}$ which are external to a given $\Lambda_{j}^{i}$ is not determined, since we find that a curve $\Lambda_{j}^{i}$ can be connected by the stable manifold $W_{C, i}^{s}$ to other curves $\tilde{\Lambda}_{j}^{i}$ through the five-dimensional space $\mathcal{M}_{C}$ which $W_{C, i}^{s}$ belongs to, and all the initial conditions in the interiors of both $\Lambda_{j}^{i}, \tilde{\Lambda}_{j}^{i}$ transit from $J$ to $S$ in the same number of revolutions around $P_{2}$. Similarly, we compute closed curves $M_{j}^{1}$, whose interior points transits from $S$ to $J$ after the same number of revolutions around $P_{1}$. The transits from $J$ to $S$ (or from $S$ to $J$ ) and from $J$ to $E$ need a passage through the isolating blocks $R_{1}, R_{2}$ respectively.

The article is structured as follows. In Section 2 we introduce the specific chaos indicators for the spatial three-body problem whose ridges approximate the hypertube manifolds; in Sections 3 we provide example of computations of the manifolds for the Sun-Jupiter mass ratio and we discuss the dynamics of orbits with initial conditions in the vertical section $\Sigma_{V}$; in Section 4 we discuss the discontinuities of chaos indicators due to the KS transfrormation; in Section 5 we provide some Conclusions.

\section{Geometric chaos indicators for the spatial restricted three- body problem}

The finite time chaos indicators, such as the finite time Lyapunov indicator (FTLE hereafter) and the fast Laypunov indicator (FLI hereafter), originally defined in [36, 7], have been used in the last decade to compute numerical approximations of the stable and unstable manifolds of equilibria, periodic orbits, and the so called Lagrangian coherent structures of turbulent flows of many model systems (see for example $[6,10,11,37,22,12,30,20,21,8,14,25]$ ). In [13] we have shown that, depending on certain non-linear properties of the dynamical system, the traditional finite time chaos indicators can fail completely the detection of the stable or 

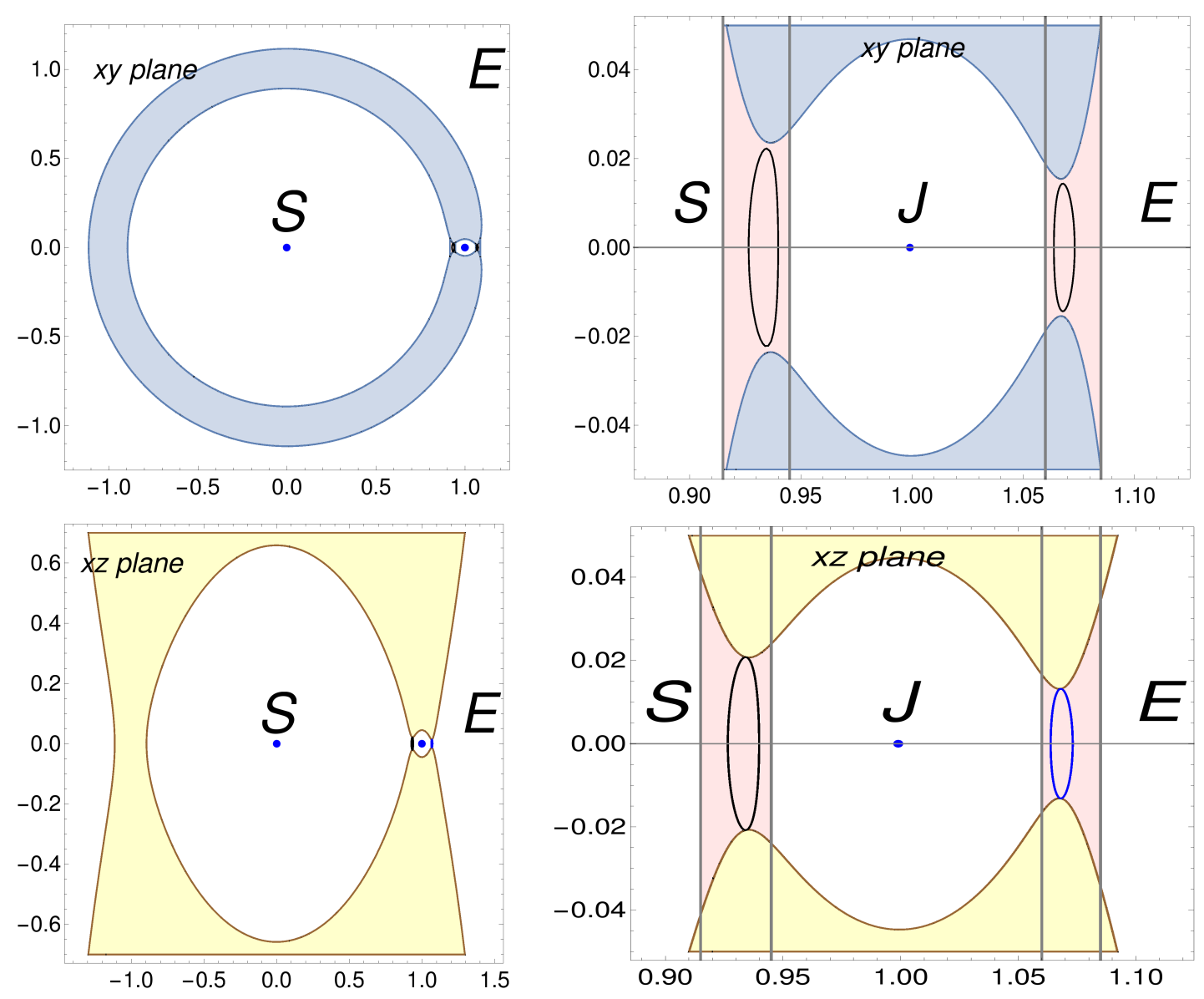

Figure 1: Representation of the realms of motion $S, J, E$ and of the isolating blocks $R_{1}, R_{2}$ in the $x, y$ plane $(z=0)$, top panels, and in the $x, z$ plane $(y=0)$, bottom panels, for a value of the Jacobi constant, slightly smaller than $C_{2}$. The right panels are obtained as a zoom at $P_{2}$ of the left panels. The vertical gray lines, located at $x=x_{1}, x_{2}, x_{3}, x_{4}$, represent the boundaries of two isolating blocks $R_{1}, R_{2}$ containing the center manifolds of $L_{1}$ and $L_{2}$ for the same value $C$ of the Jacobi constant. The black curves in the $x, y$ plane represent the horizontal Lyapunov orbits of $L_{1}, L_{2}$, while the blue curves in the $x, z$ plane represent the intersections of center manifolds of $L_{1}, L_{2}$ with the vertical section $\Sigma_{V}$ (computed as explained in Section 2).

unstable manifolds of hyperbolic equilibria or periodic orbits. The problem has been solved by taking into account for the computation of the chaos indicator only the contributions from the variational equations due to a neighbourhood of the hyperbolic fixed point or periodic orbit. The numerical computation of the stable and manifolds of the center manifolds of $L_{1}, L_{2}$ in the spatial restricted three-body problem needs major modifications of the method used for 
the planar problem $[13,24]$ for three reasons: (i) the center manifold $W_{C, i}^{s}$ has dimension 3 in a 5-dimensional reduced phase-space (while in the planar problem is just a periodic orbit in a 3 dimensional reduced phase-space) and (ii) does not contain only periodic or quasi-periodic orbits; (iii) the Kustaanheimo-Stiefel regularization of the spatial problem is geometrically more complicate than the planar Levi-Civita regularization. These problems are solved by considering a family of modified chaos indicators, which are introduced in the next subsections.

\subsection{The KS transformation}

The Kustaanheimo-Stiefel regularization at the secondary body $P_{2}$ is defined by the introduction of redundant spatial variables $u=\left(u_{1}, u_{2}, u_{3}, u_{4}\right)$ related to the Cartesian variables $(x, y, z)$ by:

$$
\left\{\begin{array}{l}
x=1-\mu+u_{1}^{2}-u_{2}^{2}-u_{3}^{2}+u_{4}^{2} \\
y=2\left(u_{1} u_{2}-u_{3} u_{4}\right) \\
z=2\left(u_{1} u_{3}+u_{2} u_{4}\right)
\end{array}\right.
$$

and by the time transformation:

$$
d t=r_{2} d s
$$

where $t$ denotes the physical time, and $s$ is called the proper time. The map defined by equations (6) will be denoted by:

$$
\begin{aligned}
\pi: \mathbb{U} & \longrightarrow \mathbb{R}^{3} \\
\left(u_{1}, u_{2}, u_{3}, u_{4}\right) & \longmapsto \pi\left(u_{1}, u_{2}, u_{3}, u_{4}\right)=(x, y, z),
\end{aligned}
$$

where $\mathbb{U}$ denotes the space of the $u=\left(u_{1}, u_{2}, u_{3}, u_{4}\right)$ variables. As usual, we also find convenient to consider the four dimensional extension of $(8)$ :

$$
\begin{gathered}
\tilde{\pi}: \mathbb{U} \longrightarrow \mathbb{R}^{4} \\
u \longmapsto(x, y, z, q)=A(u) u
\end{gathered}
$$

with:

$$
A(u)=\left(\begin{array}{cccc}
u_{1} & -u_{2} & -u_{3} & u_{4} \\
u_{2} & u_{1} & -u_{4} & -u_{3} \\
u_{3} & u_{4} & u_{1} & u_{2} \\
u_{4} & -u_{3} & u_{2} & -u_{1}
\end{array}\right) .
$$

For a fixed value $C$ of the Jacobi constant, the equations of motion of the spatial restricted three-body problem in the variables $u$ and proper time $s \operatorname{are}^{3}$ (see, for example, [5]):

$$
\left\{\begin{array}{l}
u_{1}^{\prime \prime}=\left(K_{1}+K_{2}\right) u_{1}+K_{3} u_{2}-K_{4} u_{3} \\
u_{2}^{\prime \prime}=\left(K_{1}-K_{2}\right) u_{2}+K_{3} u_{1}-K_{4} u_{4} \\
u_{3}^{\prime \prime}=\left(K_{1}-K_{2}\right) u_{3}-K_{3} u_{4}-K_{4} u_{1} \\
u_{4}^{\prime \prime}=\left(K_{1}+K_{2}\right) u_{4}-K_{3} u_{3}-K_{4} u_{2}
\end{array}\right.
$$

\footnotetext{
${ }^{3}$ The primes denote derivatives with respect to the proper time $s$.
} 
with $K_{j}\left(u, u^{\prime}\right), j=1, \ldots, 4$, regular functions at $u=0$, complemented with the non holonomic constraint:

$$
u \cdot \Omega u^{\prime}=0 \quad, \quad \Omega=\left(\begin{array}{cccc}
0 & 0 & 0 & 1 \\
0 & 0 & -1 & 0 \\
0 & 1 & 0 & 0 \\
-1 & 0 & 0 & 0
\end{array}\right) .
$$

In the following, we denote by $\xi=\left(x, y, z, v_{x}, v_{y}, v_{z}\right) \in T \mathbb{R}^{3}$ the Cartesian phase-space variables, by $\zeta=(u, \nu) \in T \mathbb{U}=\mathbb{R}^{8}$ the Kustaanheimo Stiefel phase-space variables and by:

$$
\begin{gathered}
\mathcal{X}: T \mathbb{U}_{0} \longrightarrow T \mathbb{R}^{3} \\
\zeta \longmapsto \xi=\mathcal{X}(\zeta)
\end{gathered}
$$

the projection:

$$
\begin{aligned}
(x, y, z) & =\pi(u) \\
\left(v_{x}, v_{y}, v_{z}\right) & =\frac{2}{\|u\|^{2}} \frac{\partial \pi}{\partial u} \nu
\end{aligned}
$$

where $^{4} \mathbb{U}_{0}=\mathbb{U} \backslash 0$.

The solutions of equations (11) project on the solutions of equations (1) in the following sense (again, for the details, see [5]): consider a solution $u(s)$ of equations (11), with initial conditions $\left(u(0), u^{\prime}(0)\right)$ satisfying equations (12), defined in some neighbourhood $\mathcal{S}$ of $s=0$ with $\pi(u(s)) \neq(-\mu, 0,0),(1-\mu, 0,0)$ for all $s \in \mathcal{S}$; denote by:

$$
t(s)=\int_{0}^{s}\|u(\sigma)\|^{2} d \sigma
$$

and consider the solution $(x(t), y(t), z(t))$ of (1) of initial conditions: $(x(0), y(0), z(0), \dot{x}(0), \dot{y}(0), \dot{z}(0))=$ $\chi\left(u(0), u^{\prime}(0)\right)$. Then, we have: $(x(t(s)), y(t(s)), z(t(s)))=\pi(u(s))$ for all $s \in \mathcal{S}$.

The variational equations of the regularized equations of motion (11) are obtained from the first order differential equations:

$$
\left\{\begin{array}{l}
u_{i}^{\prime}=\nu_{i}, i=1, \ldots, 4 \\
\nu_{1}^{\prime}=\left(K_{1}+K_{2}\right) u_{1}+K_{3} u_{2}-K_{4} u_{3} \\
\nu_{2}^{\prime}=\left(K_{1}-K_{2}\right) u_{2}+K_{3} u_{1}-K_{4} u_{4} \\
\nu_{3}^{\prime}=\left(K_{1}-K_{2}\right) u_{3}-K_{3} u_{4}-K_{4} u_{1} \\
\nu_{4}^{\prime}=\left(K_{1}+K_{2}\right) u_{4}-K_{3} u_{3}-K_{4} u_{2}
\end{array}\right.
$$

denoted in compact form by:

$$
\zeta^{\prime}=F_{C}(\zeta)
$$

\footnotetext{
${ }^{4}$ Since we are interested only in the solutions $u(s)$ of $(11)$ which project to solutions of (1), the restriction to $\mathbb{U}_{0}$ is allowed.
} 
For any solution $\zeta(s)$ of (16) we define the variational equations:

$$
w^{\prime}=\frac{\partial F_{C}}{\partial \zeta}(\zeta(s)) w
$$

where $w=\left(w_{u}, w_{\nu}\right) \in T T \mathbb{U}$ is a vector tangent to the domain of the Kustaanheimo Stiefel phase-space variables $\zeta$.

Remark 1. In Section 4 we will show that the solutions of the variational equations (17) do not project on the solutions of the variational equations of the non-regularized equations of motion (1). This apparent contradiction is due to the fact that the Kustaanheimo Stiefel transformation, including the proper time transformation, is not simply a transformations of variables and therefore the usual conjugation of variational equations under smooth changes of variables does not apply. In particular, the chaos indicators constructed from the solutions of the variational equations, such as the Lyapunov exponents, the fast Lyapunov indicators, the finite time Lyapunov exponents, are different if they are defined from the solutions of (17) or from the solutions of variational equations of the non-regularized equations of motion (1). We are therefore faced with a choice: is it more convenient to define chaos indicators from the smooth variational equations (17) or from the singular variational equations obtained in the Cartesian variables? The choice of using the chaos indicators defined by the smooth variational equations (17) appears as mandatory. In fact, the numerical integration of the singular variational equations introduces very strong numerical instabilities ${ }^{5}$ already in the planar case (see [23]), which instead are completely under control using equations (17). On the other hand, as we will see below, the FLI defined using the evolution of the tangent vectors in the KS variables have discontinuities.

\subsection{Geometric chaos indicators for the spatial restricted three-body problem}

The solutions $w(s)$ of the variational equations (17) enter the definition of the chaos indicators which we use to compute the hypertube manifolds of the spatial restricted three-body problem. Through all this section, by $\phi$ we denote any fixed non negative smooth function:

$$
\phi: \mathcal{M}_{C} \longrightarrow[0,+\infty) \text {. }
$$

Definition 1. For any $\xi_{0}=\left(x_{0}, y_{0}, z_{0}, \dot{x}_{0}, \dot{y}_{0}, \dot{z}_{0}\right) \in \mathcal{M}_{C}$ we consider a smooth pre-image of $\chi$ :

$$
\zeta=\chi^{-1}(\xi)=\left(\pi^{-1}(x, y, z), \tilde{\pi}^{-1}(\xi)\right)
$$

defined in a neighbourhood $\mathcal{W}$ of $\xi_{0}$. Then, for any positive time $T>0$, we define the modified finite time Lyapunov exponent (mFTLE hereafter):

$$
\mathcal{F}_{\phi}^{T}: \mathcal{W} \longrightarrow \mathbb{R}
$$

\footnotetext{
${ }^{5}$ The variational equations (51) have singularities of higher order with respect to the singularities of the equations of motion, since they are obtained by further differentiating the singular terms $1 / r_{1}^{3}, 1 / r_{2}^{3}$.
} 
by:

$$
\mathcal{F}_{\phi}^{T}(\xi)=\max _{t \in[0, T]} \max _{w_{0} \in \mathbb{R}^{8},\left\|w_{0}\right\|=1} \int_{0}^{\sigma(\xi, t)} \phi\left(\mathcal{X}(\zeta(s)) \frac{w(s) \cdot w^{\prime}(s)}{\|w(s)\|^{2}} d s .\right.
$$

where $\zeta(s)$ is the solution of equations (16) with initial condition $\zeta(0)=\chi^{-1}(\xi) ; w(s)$ is the solution of the variational equations (17) defined by $\zeta(s)$ with initial tangent vector $w(0)=w_{0}$; $\sigma(\xi, t)$ denotes the transformation $s:=\sigma(\xi, t)$ between the physical time $t$ and the proper time $s$ for the orbit with initial conditions $\xi$.

Equation (19) provides a local definition for the modified FTLE, since it depends on the specific choice of the local inversion function $\chi^{-1}$. Since we have to compute the chaos indicators in extended domains of the phase-space, we need to consider global extensions. A non trivial property of the function defined in (19) is that its values are independent on the specific choices of $\chi^{-1}$, In fact, we have the following

Theorem 1. For any value $C$ of the Jacobi constant and for any $T>0$, consider any open set $\mathcal{M}_{C, T} \subseteq \mathcal{M}_{C}$ such that the solution $(x(t), y(t), z(t))$ of (1) with initial conditions $(x(0), y(0), z(0), \dot{x}(0), \dot{y}(0), \dot{z}(0)) \in \mathcal{M}_{C, T}$ exist with $(x(t), y(t), z(t)) \in \mathbb{R}_{0}^{3}$ for all $t \in[0, T]$. Then, any local definition of the modified finite fime Lyapunov exponent obtained from (19) has a smooth global extension to $\mathcal{M}_{C, T}$.

The proof of theorem 1 is reported in Section 4. Here, we remark that the existence of smooth global extensions of (19) is obtained from the computation of the maximum over all the tangent vectors of unitary norm. Since the computation of the maximum is CPU-expensive, it is convenient to consider indicators defined by integrating single initial tangent vectors, in the spirit of the definition of Fast Lyapunov Indicators originally introduced in [7]. Since the Fast Lyapunov Indicators cannot be globally extended to $\mathcal{M}_{C, T}$, we need to consider at least two indicators defined using an atlas of two local inversions $\chi_{-}^{-1}, \chi_{+}^{-1}$ of the KS transformation:

$$
\begin{aligned}
& \pi_{-}^{-1}: \mathbb{R}^{3} \backslash\{(x, 0,0): x \geq 1-\mu\} \longrightarrow \mathbb{U} \\
& \pi_{+}^{-1}: \mathbb{R}^{3} \backslash\{(x, 0,0): x \leq 1-\mu\} \longrightarrow \mathbb{U}
\end{aligned}
$$

defined by

$$
\begin{aligned}
& \pi_{-}^{-1}(x, y, z)=\left(\frac{y}{\sqrt{2(r-X)}}, \frac{\sqrt{r-X}}{\sqrt{2}}, 0, \frac{z}{\sqrt{2(r-X)}}\right) \\
& \pi_{+}^{-1}(x, y, z)=\left(\frac{\sqrt{r+X}}{\sqrt{2}}, \frac{y}{\sqrt{2(r+X)}}, \frac{z}{\sqrt{2(r+X)}}, 0\right),
\end{aligned}
$$

where $X=x-1+\mu, r=\sqrt{X^{2}+y^{2}+z^{2}}$. These maps are smooth and satisfy $\pi \circ \pi_{-}^{-1}(x, y, z)=$ $(x, y, z), \pi \circ \pi_{+}^{-1}(x, y, z)=(x, y, z)$. The local inversions $\chi_{-}^{-1}, \chi_{+}^{-1}$ of $\chi$ are constructed by 
extending the inverse maps $\pi_{-}^{-1}, \pi_{+}^{-1}$ to the velocities:

$$
(u, v)=\chi_{ \pm}^{-1}(x, y, z, \dot{x}, \dot{y}, \dot{z})=\left(\pi_{ \pm}^{-1}(x, y, z), \tilde{\pi}_{ \pm}^{-1}(x, y, z, \dot{x}, \dot{y}, \dot{z})\right)
$$

with $\tilde{\pi}_{-}^{-1}, \tilde{\pi}_{+}^{-1}$ defined by:

$$
\tilde{\pi}_{ \pm}^{-1}(x, y, z, \dot{x}, \dot{y}, \dot{z})=\frac{1}{2} A^{T}\left(\pi_{ \pm}^{-1}(x, y, z)\right)(\dot{x}, \dot{y}, \dot{z}, 0) .
$$

Now we can define two fast Lyapunov indicators whose joint domain span all the possible initial conditions of the Cartesian phase-space.

Definition 2. For any positive time $T>0$, for any $\xi_{0}=\left(x_{0}, y_{0}, z_{0}, \dot{x}_{0}, \dot{y}_{0}, \dot{z}_{0}\right) \in \mathcal{M}_{C}$ and any $w_{0} \in \mathbb{R}^{8}$, if $\left(x_{0}, y_{0}, z_{0}\right) \in \mathbb{R}^{3} \backslash\{(x, 0,0): x \leq 1-\mu\}$ we define the modified fast Lyapunov indicator (mFLI hereafter) by:

$$
F L I_{\phi}^{+}\left(\xi_{0}, w_{0}, T\right)=\max _{t \in[0, T]} \int_{0}^{\sigma\left(\xi_{0}, t\right)} \phi\left(\mathcal{X}\left(\zeta_{+}(s)\right) \frac{w_{+}(s) \cdot w_{+}^{\prime}(s)}{\left\|w_{+}(s)\right\|^{2}} d s\right.
$$

while if $\left(x_{0}, y_{0}, z_{0}\right) \in \mathbb{R}^{3} \backslash\{(x, 0,0): x>1-\mu\}$ we define the modified fast Lyapunov indicator (mFLI hereafter) by:

$$
F L I_{\phi}^{-}\left(\xi_{0}, w_{0}, T\right)=\max _{t \in[0, T]} \int_{0}^{\sigma\left(\xi_{0}, t\right)} \phi\left(\mathcal{X}\left(\zeta_{-}(s)\right) \frac{w_{-}(s) \cdot w_{-}^{\prime}(s)}{\left\|w_{-}(s)\right\|^{2}} d s\right.
$$

where $\zeta_{ \pm}(s)$ are the solutions of equations (16) with initial conditions $\zeta_{ \pm}(0)=\chi_{ \pm}^{-1}\left(\xi_{0}\right) ; w_{ \pm}(s)$ is the solution of the variational equations (17) defined by $\zeta_{ \pm}(s)$ with initial conditions $w_{ \pm}(0)=w_{0}$ and $\sigma\left(\xi_{0}, t\right)$ denotes the the transformation $s:=\sigma\left(\xi_{0}, t\right)$ between the physical time $t$ and the proper time $s$ for the orbit with initial condition $\xi_{0}$.

Remark 2. For any fixed $w_{0} \in \mathbb{R}^{8}$, and if $\left(y_{0}, z_{0}\right) \neq(0,0)$, both indicators $F L I_{\phi}^{-}, F L I_{\phi}^{+}$are defined, and the correspondence between them is:

$$
F L I_{\phi}^{+}\left(\xi_{0}, w_{0}, T\right)=F L I_{\phi}^{-}\left(\xi_{0}, R_{\alpha} w_{0}, T\right)
$$

where $R_{\alpha} \zeta=\left(\mathcal{R}_{\alpha} u, \mathcal{R}_{\alpha} v\right)$ with:

$$
\mathcal{R}_{\alpha}=\left(\begin{array}{cccc}
\cos \alpha & 0 & 0 & -\sin \alpha \\
0 & \cos \alpha & \sin \alpha & 0 \\
0 & -\sin \alpha & \cos \alpha & 0 \\
\sin \alpha & 0 & 0 & \cos \alpha
\end{array}\right),
$$

and the value of $\alpha$ is defined by $\chi_{-}^{-1}\left(\xi_{0}\right)=R_{\alpha} \chi_{+}^{-1}\left(\xi_{0}\right)$ (see Section 4 for the details). 
Remark 3. If instead of the finite time chaos indicators we consider the Lyapunov exponents defined using the regularized variational equations (17):

$$
l_{ \pm}\left(\xi_{0}, w_{0}\right)=\lim _{s \rightarrow+\infty} \frac{1}{s} \ln \left\|w_{ \pm}(s)\right\|
$$

where $w_{ \pm}(s)$ denote the solutions of (17) defined by the solutions $\zeta_{ \pm}(s)$ of (16) with initial conditions $\zeta_{ \pm}(0)=\chi_{ \pm}^{-1}\left(\xi_{0}\right)$, we have:

$$
l_{+}\left(\xi_{0}, w_{0}\right)=l_{-}\left(\xi_{0}, R_{\alpha} w_{0}\right)
$$

where $\alpha$ is defined by $\chi_{-}^{-1}\left(\xi_{0}\right)=R_{\alpha} \chi_{+}^{-1}\left(\xi_{0}\right)$. Again, the largest Lyapunov exponent:

$$
l\left(\xi_{0}\right)=\max _{w_{0} \in \mathbb{R}^{8}:\left\|w_{0}\right\|=1} l_{+}\left(\xi_{0}, w_{0}\right)=\max _{w_{0} \in \mathbb{R}^{8}:\left\|w_{0}\right\|=1} l_{-}\left(\xi_{0}, w_{0}\right)
$$

is globally defined (if the limits converge to some real values), while the individual Lyapunov exponents $l_{ \pm}\left(\cdot, w_{0}\right)$ are not.

Remark 4. In $[12,24]$ we defined modified FLIs by using the variational equations of the planar circular restricted three-body problem regularized with the Levi-Civita transformation:

$$
\left\{\begin{array}{l}
x=1-\mu+u_{1}^{2}-u_{2}^{2} \\
y=2 u_{1} u_{2}
\end{array}\right.
$$

and by the proper-time transformation $d t=r_{2} d s$. For $(x, y) \neq(1-\mu, 0)$ the LC transformation has two preimages $\left(u_{1}, u_{2}\right)$ and $\left(-u_{1},-u_{2}\right)$. Therefore, since the Lyapunov exponents and the fast Lyapunov indicators are defined using norms of tangent vectors, they are globally defined. The geometric problem appears only for the KS transformation.

We finally comment the modification of the FTLE (19) and FLI (22),(23) by the introduction of the function $\phi(\mathcal{X}(\zeta(s))$ in the integrand which weights the contribution to the indicator of the solutions $w(s)$ of the variational equations. For the special choice

$$
\phi(\xi)=1 \quad \forall \xi \in \mathcal{M}_{C},
$$

the mFLI defined in (22), (23) are the usual fast Lyapunov indicators introduced in [7] defined using the regularized variational equations (17):

$$
F L I_{\phi}^{ \pm}\left(\xi_{0}, w_{0}, T\right)=\max _{t \in[0, T]} \ln \frac{\left\|w_{ \pm}\left(\sigma\left(\xi_{0}, t\right)\right)\right\|}{\left\|w_{0}\right\|}
$$

and the mFTLE becomes:

$$
\mathcal{F}_{\phi}^{T}\left(\xi_{0}\right)=\max _{t \in[0, T]} \max _{w} \ln \frac{\left\|w\left(\sigma\left(\xi_{0}, t\right)\right)\right\|}{\|w(0)\|},
$$


(the quantity following the $\max _{t}$ being the usual finite time Lyapunov exponent introduced in [36], defined using the regularized variational equations (17)). Instead, when the weight function $\phi(\xi)$ is equal (or very close) to 1 in a small neighbourhood $\mathcal{U}_{i}$ of the center manifold $W_{C, i}^{c}$, and rapidly decays to zero outside $\mathcal{U}_{i}$, computations of the indicators $F L I_{\phi}^{ \pm}$and $\mathcal{F}_{\phi}^{T}$ provide sharp localization of the stable manifolds $W_{C, i}^{s}$ of $W_{C, i}^{c}$ (the unstable manifolds are obtained by computing the indicators for negative times $T$ ), as it will be explained in the nex Subsection.

\subsection{Hypertube manifolds computations with the geometric chaos indicators}

Several definitions of ridges for finite time chaos indicators have been given in the literature since $[21,30,26]$, corresponding to the motivations related to the definition of the so called Lagrangian coherent structures. In this paper, the ridges of the chaos indicators are used with different purposes, for which we provide the following definition.

Definition 3. Let us consider a two dimensional real function $F: \mathbb{R}^{2} \rightarrow \mathbb{R}$. The smooth curve $\Gamma \in \mathbb{R}^{2}$ is a ridge of $F$ if there exists a family of curves $\gamma_{\eta}(x)$ (x, defined in a neighbourhood of 0 , is a parameter along the curve; $\eta$ is defined in an interval) transverse to $\Gamma$ at $\gamma_{\eta}(0)$, and such that $F\left(\gamma_{\eta}(x)\right)$ has a strict absolute maximum at $x=0$.

Specifically, we will consider the ridges of the modified chaos indicators (19), (22), (23) restricted to two-dimensional surfaces $\Sigma \subseteq \mathcal{M}_{C}$ transverse to the stable manifolds $W_{C, i}^{s}$. We claim that for suitable choices of the neighbourhood $\mathcal{U}_{i}$, of the weight function $\phi(\xi)$ and, for the $F L I_{\phi}^{ \pm}$of the parameters $w_{0}$, the subset $W^{s}(T) \cap \Sigma$ of the stable manifolds defined by:

$$
W^{s}(T)=\left\{\xi \in W_{C, i}^{s}: \quad \varphi(t, \xi) \in W_{C, l o c}^{s} \forall t \geq T / 2\right\}
$$

where $\varphi(t, \xi)$ denotes the flow of the three-body problem in the Cartesian phase-space and

$$
W_{C, l o c}^{s}=\left\{\xi \in \mathcal{U}_{i} \cap W_{C, i}^{s}: \quad \varphi(t, \xi) \in \mathcal{U}_{i} \cap W_{C, i}^{s} \forall t \geq 0\right\}
$$

denotes the local stable manifold in the set $\mathcal{U}_{i}$, is close to a ridge of the indicators $F L I_{\phi}^{ \pm}\left(\xi, w_{0}, T\right), \mathcal{F}_{\phi}^{T}(\xi)$ restricted to $\Sigma$.

Let us continue our discussion by considering the case of $L_{1}$ (the case of $L_{2}$ can be obtained with very simple modifications) and by fixing the total time $T$ and a value $C$ of the Jacobi constant; we will assume, where needed, that $\varepsilon=C-C_{1}>0$ is suitably small. While in Definitions 1 and 2 (as well as in the applications of Section 3) the weight function $\phi(\xi)$ is smooth, in this Section we consider for simplicity a weight function $\phi(\xi)$ corresponding to the characteristic function of a suitably small neighbourhood $\mathcal{U}_{i}$ of $W_{C, i}^{c}$. Similar results are obtained for smooth weight functions whose value is 1 in the set $\mathcal{U}_{i}$, and outside $\mathcal{U}_{i}$ rapidly decay to zero. We consider two properties of the dynamics in the sets $\mathcal{U}_{i}$ which are valid with the above assumptions. 
- The scattering time from $\mathcal{U}_{i}$. Any solution with initial conditions $\xi \in \mathcal{U}_{i} \backslash W_{C, l o c}^{s}$ is scattered outside $\mathcal{U}_{i}$ in a finite time $t_{\xi}$ (i.e. there exists a minimum $t_{\xi}>0$ such that $\varphi\left(t_{\xi}, \xi\right) \notin \mathcal{U}_{i}$ ). The exit time $t_{\xi}$ diverges at the local stable manifold.

- Expansions of tangent vectors in $\mathcal{U}_{i}$. For any $\zeta$ such that $\chi(\zeta) \in \mathcal{U}_{i}$, and any $s>0$, we consider:

$$
\lambda(s, \zeta)=\frac{1}{s} \ln \left\|\frac{\partial \tilde{\varphi}}{\partial \zeta}(s, \zeta)\right\|,
$$

where $\tilde{\varphi}(s, \zeta)$ denotes the flow of $(16)^{6}$ In the above hypotheses, $\lambda(s, \zeta)$ is continuous with respect to $\zeta$; also, for any $\zeta$ such that $\chi(\zeta) \in \mathcal{U}_{i}$ and any $s \in[0, S(\zeta, T)]$ with:

$$
S(\zeta, T)=\min \left(\sigma(\chi(\zeta), T), \sup \left\{s \geq 0: \quad \chi(\tilde{\varphi}(s, \zeta)) \in \mathcal{U}_{i}\right\}\right),
$$

we have

$$
0<\lambda_{\varepsilon}^{1} \leq \lambda(s, \zeta) \leq \lambda_{\varepsilon}^{2}
$$

with $\lambda_{\varepsilon}^{1}, \lambda_{\varepsilon}^{2}$ constants depending only on $\varepsilon$ (and the fixed $T$ ) and satisfying:

$$
\lim _{\varepsilon \rightarrow 0} \lambda_{\varepsilon}^{1}=\lim _{\varepsilon \rightarrow 0} \lambda_{\varepsilon}^{2}=\lambda^{\varepsilon},
$$

with $\lambda^{\varepsilon}$ defined from the linearizations of the regularized equations of motion at the Lagrange equilibrium (for details see the technical Subsection 4.3).

Below we provide estimates for $\mathcal{F}_{\phi}^{T}(\xi)$ in three different cases.

(i) Let us consider $\xi_{0} \in W_{C}^{s}(T) \cap \Sigma$. Denote by $T_{\xi_{0}}<T / 2$ the minimum time $t$ such that $\varphi\left(\xi_{0}, t\right) \in \overline{W_{C, l o c}^{s}}$, for any $t \in\left[T_{\xi_{0}}, T\right]$.

For any solution $\zeta(s)$ of equations (16) with $\chi(\zeta(s))=\varphi\left(t(s), \xi_{0}\right)$ and any solution $w(s)$ of the variational equations (17) defined by $\zeta(s)$ with $w(0)=w_{0}$, we have:

$$
\int_{0}^{\sigma\left(\xi_{0}, t\right)} \phi\left(\varphi\left(t(s), \xi_{0}\right)\right) \frac{w(s) \cdot w^{\prime}(s)}{\|w(s)\|^{2}} d s=\ln \frac{\left\|w\left(\sigma\left(\xi_{0}, t\right)\right)\right\|}{\left\|w\left(\sigma\left(\xi_{0}, T_{\xi_{0}}\right)\right)\right\|}, \forall t \geq T_{\xi_{0}} .
$$

Since $\tilde{\varphi}(s, \cdot)$ is a diffeomorphism, from (30) and (29) we obtain:

$$
\begin{gathered}
\mathcal{F}_{\phi}^{T}\left(\xi_{0}\right)=\max _{t \in[0, T]} \max _{w_{0} \in \mathbb{R}^{8},\left\|w_{0}\right\|=1} \int_{0}^{\sigma\left(\xi_{0}, t\right)} \phi\left(\varphi\left(t(s), \xi_{0}\right)\right) \frac{w(s) \cdot w^{\prime}(s)}{\|w(s)\|^{2}} d s= \\
=\max _{t \in[0, T]} \max _{w_{0} \in \mathbb{R}^{8},\left\|w_{0}\right\|=1} \ln \left\|\frac{\partial \tilde{\varphi}}{\partial \zeta}\left(\sigma\left(\xi_{0}, t\right)-\sigma\left(\xi_{0}, T_{\xi_{0}}\right), \zeta\left(\sigma\left(\xi_{0}, T_{\xi_{0}}\right)\right)\right) w_{0}\right\| \\
=\max _{t \in[0, T]} \ln \left\|\frac{\partial \tilde{\varphi}}{\partial \zeta}\left(\sigma\left(\xi_{0}, t\right)-\sigma\left(\xi_{0}, T_{\xi_{0}}\right), \zeta\left(\sigma\left(\xi_{0}, T_{\xi_{0}}\right)\right)\right)\right\| \\
=\max _{t \in[0, T]}\left(\sigma\left(\xi_{0}, t\right)-\sigma\left(\xi_{0}, T_{\xi_{0}}\right)\right) \lambda\left(\sigma\left(\xi_{0}, t\right), \zeta\left(\sigma\left(\xi_{0}, T_{\xi_{0}}\right)\right)\right) \\
\in\left(\sigma\left(\xi_{0}, T\right)-\sigma\left(\xi_{0}, T_{\xi_{0}}\right)\right)\left[\lambda_{\varepsilon}^{1}, \lambda_{\varepsilon}^{2}\right] .
\end{gathered}
$$

\footnotetext{
${ }^{6}$ The norm of the Jacobian matrix is the matrix norm: $\|B\|=\max _{V:\|V\|=1}\|B V\|$.
} 
(ii) Let us consider $\xi \notin W_{C}^{s}(T)$, but very close to $\xi_{0}$. Denote by $T_{\xi}$ the minimum time such that $\varphi(t, \xi) \in \overline{\mathcal{U}}_{i}$. For any solution $\zeta(s)$ of equations (16) with $\chi(\zeta(s))=\varphi(t(s), \xi)$ we denote by $w(s)$ the solution of the variational equations (17) defined by $\zeta(s)$ with $w(0)=w_{0}$. From standard Lipschitz estimates we have that $T_{\xi}$ is close to $T_{\xi_{0}}$, and for suitably small $\left\|\xi-\xi_{0}\right\|$, we have $\varphi(t, \xi) \in \mathcal{U}_{i}$ for any $t \in\left[T_{\xi}, T\right]$. The estimate of the FTLE is therefore

$$
\begin{gathered}
\mathcal{F}_{\phi}^{T}(\xi)=\max _{t \in[0, T]} \max _{w_{0} \in \mathbb{R}^{8},\left\|w_{0}\right\|=1} \int_{0}^{\sigma(\xi, t)} \phi(\varphi(t(s), \xi)) \frac{w(s) \cdot w^{\prime}(s)}{\|w(s)\|^{2}} d s= \\
=\max _{t \in[0, T]} \ln \left\|\frac{\partial \tilde{\varphi}}{\partial \zeta}\left(\sigma(\xi, t)-\sigma\left(\xi, T_{\xi}\right), \zeta\left(\sigma\left(\xi, T_{\xi}\right)\right)\right)\right\| \\
=\max _{t \in[0, T]}\left(\sigma(\xi, t)-\sigma\left(\xi, T_{\xi}\right)\right) \lambda\left(\sigma(\xi, t), \zeta\left(\sigma\left(\xi, T_{\xi}\right)\right)\right) \\
\in\left(\sigma(\xi, T)-\sigma\left(\xi, T_{\xi}\right)\right)\left[\lambda_{\varepsilon}^{1}, \lambda_{\varepsilon}^{2}\right] .
\end{gathered}
$$

(iii) Let us consider again $\xi \notin W_{C}^{s}(T)$ so close to $\xi_{0}$ that $T_{\xi}$ is close to $T_{\xi_{0}}$ (as in case (ii)), but now assume that the scattering time $t_{\varphi\left(T_{\xi}, \xi\right)}$ of $\varphi\left(T_{\xi}, \xi\right)$ satisfies:

$$
t_{\varphi\left(T_{\xi}, \xi\right)} \leq T-T_{\xi_{0}}
$$

so that in the time interval $\left[T_{\xi}, T\right]$ the solution $\varphi(t, \xi)$ exits from $\mathcal{U}_{i}$. We consider the sequence of intervals for the proper time $s$ :

$$
I_{1}=\left(a_{1}, b_{1}\right), I_{2}=\left(a_{2}, b_{2}\right), \ldots, I_{M_{\xi}}=\left(a_{M_{\xi}}, b_{M_{\xi}}\right) \subseteq\left[\sigma\left(T_{\xi}, \xi\right), \sigma(T, \xi)\right]
$$

defined by $a_{1}<b_{1} \leq a_{2}<b_{2} \leq \ldots<b_{M_{\xi}}$ such that $\chi(\zeta(s)) \in \mathcal{U}_{i}$ if and only if $s \in \cup I_{j}$. We have

$$
\begin{gathered}
\mathcal{F}_{\phi}^{T}(\xi)=\max _{t \in[0, T]} \max _{w_{0} \in \mathbb{R}^{8},\left\|w_{0}\right\|=1} \int_{0}^{\sigma(\xi, t)} \phi(\varphi(t(s), \xi)) \frac{w(s) \cdot w^{\prime}(s)}{\|w(s)\|^{2}} d s= \\
\leq \sum_{j=1}^{M_{\xi}}\left|\ln \left\|\frac{\partial \tilde{\varphi}}{\partial \zeta}\left(b_{j}-a_{j}, \zeta\left(a_{j}\right)\right)\right\|\right| \\
\leq \sum_{j=1}^{M_{\xi}}\left(b_{j}-a_{j}\right) \lambda\left(b_{j}-a_{j}, \frac{\partial \tilde{\varphi}}{\partial \zeta}\left(b_{j}-a_{j}, \zeta\left(a_{j}\right)\right)\right) \\
\in \sum_{j=1}^{M_{\xi}}\left(b_{j}-a_{j}\right)\left[\lambda_{\varepsilon}^{1}, \lambda_{\varepsilon}^{2}\right] .
\end{gathered}
$$

It remains to construct a a family of curves $\gamma_{\eta}(x) \in \Sigma$ which identifies a ridge $\Gamma \subseteq \Sigma$ for $\mathcal{F}_{\phi}^{T}$ in the section $\Sigma$, and to show that, using estimates (31), (32) and (33), the ridge is pointwise close to a given curve $\Gamma_{0} \subseteq W^{s}(T) \cap \Sigma$.

Under mild transversality conditions between $\Sigma$ and $W^{s}(T)$, using a finite reduction to the center manifold defined in $[15,16]$ (see Subsection 2.5 for details), we construct a family of curves $\gamma_{\eta}(x)$ such that $\gamma_{\eta}(0) \in \Gamma_{0}$ and by considering $\tilde{\gamma}_{\eta}(x)=\varphi\left(T_{\gamma_{\eta}(x)}, \gamma_{\eta}(x)\right)$, if $t_{\tilde{\gamma}_{\eta}(x)} \leq T$ it satisfies:

$$
\frac{1}{\lambda_{L_{1}}+X \sqrt{\varepsilon}} \ln \frac{X \sqrt{\varepsilon}}{|x|}-\alpha_{1} \leq t_{\tilde{\gamma}(\eta, x)} \leq \frac{1}{\lambda_{L_{1}}-X \sqrt{\varepsilon}} \ln \frac{X \sqrt{\varepsilon}}{|x|}+\alpha_{1}
$$

with $\alpha_{1}, X$ positive constants not depending on $\varepsilon, x$. From inequalities (34) we find a neighbourhood $\mathcal{I}_{0}$ of $x=0$ such that $\gamma_{\eta}(x)$ is as in case (ii), and therefore $\mathcal{F}_{\phi}^{T}\left(\gamma_{\eta}(x)\right)$ is close to $\mathcal{F}_{\phi}^{T}\left(\gamma_{\eta}(0)\right)$; 
we also find a neighbourhood $\mathcal{I}_{1} \supset \mathcal{I}_{0}$ of $x=0$ such that, for $x \in \mathcal{I}_{1} \backslash \mathcal{I}_{0}, \gamma_{\eta}(x)$ are as in case (iii) with $M_{\xi}=1$. In particular, for $x \in \mathcal{I}_{1} \backslash \mathcal{I}_{0}$, we have:

$$
\mathcal{F}_{\phi}^{T}\left(\gamma_{\eta}(0)\right) \geq \mathcal{F}_{\phi}^{T}\left(\gamma_{\eta}(x)\right)+\left[\sigma\left(\xi_{0}, T\right)-\sigma\left(\xi_{0}, T_{\gamma_{\eta}(x)}\right)-\sigma\left(\gamma_{\eta}(x), t_{\tilde{\gamma}_{\eta}(x)}\right)\right] \lambda_{\varepsilon}^{1}+\ldots
$$

where the dots means terms which are as small as $\varepsilon$ and $|x|$. Therefore, the ridge defined by the absolute maxima of $\mathcal{F}_{\phi}^{T}(\gamma(\eta, x))$ is contained a a smaller neighbourhood of $x=0$, strictly contained in $\mathcal{I}_{1}$. A riparametrization of the curves $\gamma_{\eta}(x)$ which moves the origin of the parameter to the absolute maximum of the FTLE completes the construction of the family of curves identifying the ridge $\Gamma$.

Remark 5. The logarithmic dependence of the scattering time (34) from $x$ makes the distance between the ridge $\Gamma$ and the curve $\Gamma_{0} \subseteq W^{s}(T)$ extremely small. Precisely, we have a linear decrement of the mFTLE with respect to $\ln |x|$, up to the maximum value of a quantity proportional to $T-T_{\xi}-t_{\tilde{\gamma}_{\eta}(x)} \leq T-T_{\xi_{0}}$; differences of units in the mFTLE value typically determine a proportional number of precision digits in the localization of the stable manifold.

Remark 6 . If the curve $\Gamma_{0}$ is completely contained in the domain of the local inversions $\chi_{+}^{-1}$ or $\chi_{-}^{-1}$, with a very mild assumption of the initial tangent vector $w_{0}$, the curve $\Gamma_{0}$ is close to a ridge of $F L I_{\phi}^{+}$or $F L I_{\phi}^{-}$, defined by $w_{0}$. In fact, by excluding a linear subspace of $\mathbb{R}^{8}$, all the initial tangent vectors are expanded exponentially at rates between $\lambda_{\varepsilon}^{1}, \lambda_{\varepsilon}^{2}$.

\subsection{Definition of the weight function $\phi(\xi)$}

To compute the chaos indicators defined in Subection 2.2 having the properties discussed in Section 2.3, we need to define explicitly a weight function $\phi(\xi)$ whose values are equal to 1 very close to the center manifold $W_{C, i}^{c}$, and then decay rapidly to zero when the distance between $\xi$ and the center manifold of $L_{i}$ is larger than a fixed small radius $\rho$.

It is not sustainable, from a computational point of view, to define $\phi(\xi)$ as a function of the minimum distance between $\xi$ and a refined grid of points on the selected center manifold $W_{C, i}^{c}$. In fact, this would require to construct a grid of the same dimension as $W_{C, i}^{c}$ of step-size smaller than $\rho$ and to compute the distance of $\xi$ from all the points of the grid. Then, to compute the integrals defining the modified chaos indicators, such an operation must repeated thousands of times. As a matter of fact, the geometry of the phase-space close to $W_{C, i}^{c}$ allows us to define $\phi$ in a more efficient way.

At this purpose, we consider a finite order approximation of $W_{C, i}^{c}$ provided by the so called Hamiltonian reductions to the center manifold (see $[15,16]$ ). Precisely, for any integer $N$, we explicitly construct canonical variables $p, q$ defined in a neighbourhood of the origin (identified with $L_{1}$ or $L_{2}$ ), giving the Hamiltonian of the spatial circular restricted three-body problem the form:

$$
H=\lambda p_{1} q_{1}+\omega_{1} \frac{p_{2}^{2}+q_{2}^{2}}{2}+\omega_{2} \frac{p_{3}^{2}+q_{3}^{2}}{2}+K(p, q)+R(p, q),
$$


where $K$ depends on $p_{1}, q_{1}$ only through the product $p_{1} q_{1}$ and is at least of order 3 with respect to $p, q$ and $R$ is a remainder of order $N$. The finite order approximations of the center manifold are obtained by setting $R=0$ in the Hamiltonian (35): for the truncated Hamiltonian, the equations $p_{1}, q_{1}=0$ define the center manifold, the equation $q_{1}=0$ its local stable manifold, the equation $p_{1}=0$ its local unstable manifold, the action $p_{1} q_{1}$ is a constant of motion. The construction of the variables $p, q$ is explicit, and is achieved through $N-2$ hyperbolic Birkhoff steps (see $[15,16])$.

Using the variables $p, q$ we define a neighbourhood $\mathcal{U}_{i}\left(i=1\right.$ for $L_{1}, i=2$ for $\left.L_{2}\right)$ of the center manifold in $\mathcal{M}_{C}$. By denoting with

$$
(q, p):=(q(\xi), p(\xi))
$$

the transformation from the Cartesian phase-space variables to the $(q, p)$, we consider the set:

$$
\mathcal{U}_{i}=\left\{\xi \in \mathcal{M}_{C}:\left|q_{1}(\xi)\right|,\left|p_{1}(\xi)\right|<\rho\right\} .
$$

Since for the truncated Hamiltonian the center manifold is defined by $p_{1}(\xi), q_{1}(\xi)=0$, for the complete Hamiltonian system (35) (and therefore for the exact three-body problem) if $\rho$ is not too small the set $\mathcal{U}_{i}$ remains a neighbourhood of the center manifold.

In principle, one could think to work with the highest possible truncation order $N$ allowed by a certain value of the Jacobi constant $C$. This is not necessary, and also not convenient. In fact, on the one hand, we do not need the manifold to correspond exactly to the center of the neighbourhood $\mathcal{U}$; on the other hand, since the variables $q, p$ are explicitly constructed as the sum of polynomials of maximum order $N-1$ of the 6 Cartesian variables $\xi_{i}$, the numerical cost for their computation increases rapidly with $N$. We therefore have chosen $N=5$, and found no differences in the numerical computation of the stable-unstable manifolds with respect to $N=6$. The variables are constructed by implementing only 2 Birkhoff normalization steps, as described in the paper [16], and by truncating the series expansions at order 4 . Then, the weight function is defined by:

$$
\phi(\xi)=\left\{\begin{array}{cr}
1 & \text { if } \rho(\xi) \leq \frac{\rho}{2} \\
\frac{1}{2}\left[\cos \left(\left(\frac{\rho(\xi)}{\rho}-\frac{1}{2}\right) \pi\right)+1\right] & \text { if } \begin{array}{c}
\rho \\
2
\end{array} \rho(\xi) \leq \frac{3 r}{2} \\
0 & \text { if } \rho(\xi)>\frac{3 \rho}{2}
\end{array} .\right.
$$

where $\rho(\xi)=\sqrt{q_{1}(\xi)^{2}+p_{1}(\xi)^{2}}$.

In particular, we have $\phi(\xi)=1$ for $\rho(\xi) \leq \rho / 2$, and $\phi(\xi)$ decays to 0 at $\rho(\xi)=3 \rho / 2$. Since $\phi(\xi)$ is smooth, from Theorem 1 also the chaos indicator $\mathcal{F}_{\phi}^{T}$ is smooth, as well as the indicators $F L I_{\phi}^{+}, F L I_{\phi}^{-}$in the domains of $\chi_{+}^{-1}, \chi_{-}^{-1}$ respectively. 


\section{Application to the Sun-Jupiter case}

In this Section we represent the intersections of the stable manifolds $W_{C, 1}^{s}, W_{C, 2}^{s}$ of the center manifolds $W_{C, 1}^{c}, W_{C, 2}^{c}$ of $L_{1}, L_{2}$ with the planar section $\Sigma_{P}$ and the vertical section $\Sigma_{V}$, defined in (3) and (4), computed as the ridges of the chaos indicators defined in Section 2. As a model problem we consider the spatial three-body problem defined by the Sun-Jupiter masses and the value:

$$
C=3.0368573364394607
$$

of the Jacobi constant, which is slightly smaller than $C_{2}$. Sections of the realms $S, J, E$ as well as of the isolating blocks $R_{1}, R_{2}$ for such value have been represented in figure 1 .

\subsection{The planar section of the stable hypertube manifolds}

The planar section $\Sigma_{P}$ of the spatial circular restricted three-body problem is a subset of the reduced phase-space of the planar problem:

$$
\mathcal{M}_{P}=\{(x, y, z, \dot{x}, \dot{y}, \dot{z}): z=0, \dot{z}=0\} .
$$

The sets $W_{C, 1}^{c} \cap \mathcal{M}_{P}, W_{C, 2}^{c} \cap \mathcal{M}_{P}$ contain only a planar Lyapunov periodic orbit, which we denote by $L L_{1}, L L_{2}$ respectively; the manifolds $W_{C, 1}^{s} \cap \mathcal{M}_{P}, W_{C, 2}^{s} \cap \mathcal{M}_{P}$ are the tubes of orbits converging to $L L_{1}, L L_{2}$. Intersections of the tube manifolds with the planar section have been provided in $[13,24]$; in this subsection we reconsider the planar problem to support the interpretation of the results of the genuine spatial problem; in fact, the connected components of the intersections of the hypertube manifolds $W_{C, 1}^{s}, W_{C, 2}^{s}$ with the vertical section $\Sigma_{V}$ are continuations of planar homoclinic orbits; moreover, different components which are disconnected in $\Sigma_{V}$, are connected in $\mathcal{M}_{C}$ through paths contained in the planar section, specifically paths of $W_{C, 1}^{s} \cap \Sigma_{P}, W_{C, 2}^{s} \cap \Sigma_{P}$.

In the top panel of figure 2 we report the ridges of the mFLI on the section $\Sigma_{P}$, computed using a weight function $\phi(\xi)$ which is different from zero only in a small neighbourhood of $L L_{1}$; in the realm $J$ we have 4 ridges, which we denote by $\lambda_{1}^{1}, \ldots, \lambda_{4}^{1}$. The ridge $\lambda_{1}^{1}$, intersects $L L_{1}$ and extends on both sides of the Lyapunov orbit; all the orbits with initial conditions on $\lambda_{1}^{1}$ converge directly to $L L_{1}$ without performing circulations around $P_{1}, P_{2}$. The dynamics of orbits with initial conditions on $\lambda_{2}^{1}, \ldots, \lambda_{4}^{1}$ is more complicate. Let us consider the tube of orbits generated by the flow of the three-body problem with initial conditions on $\lambda_{2}^{1}$ (the red curve in the top panel of figure 2), represented in figure 3. The orbits obtained by integrating the initial conditions on $\lambda_{2}^{1}$ forward in time perform half circulation around $P_{2}$ and then intersect the section $\Sigma_{P}$ at some point of $\lambda_{1}^{1}$ (see figure 3, top panel); then, as all the orbits with initial conditions on $\lambda_{1}^{1}$, they converge directly to $L L_{1}$. The tube of orbits obtained by integrating the initial conditions on $\lambda_{2}^{1}$ backward in time, after half a circulation of $P_{2}$, is so stretched that a part transits directly to the realm of motions $S$ without intersecting $\Sigma_{P}$; another part transits to the realm of motions $S$ after two intersections with $\lambda_{3}^{1}$ (one with positive and one with negative $\dot{y}$ ); 

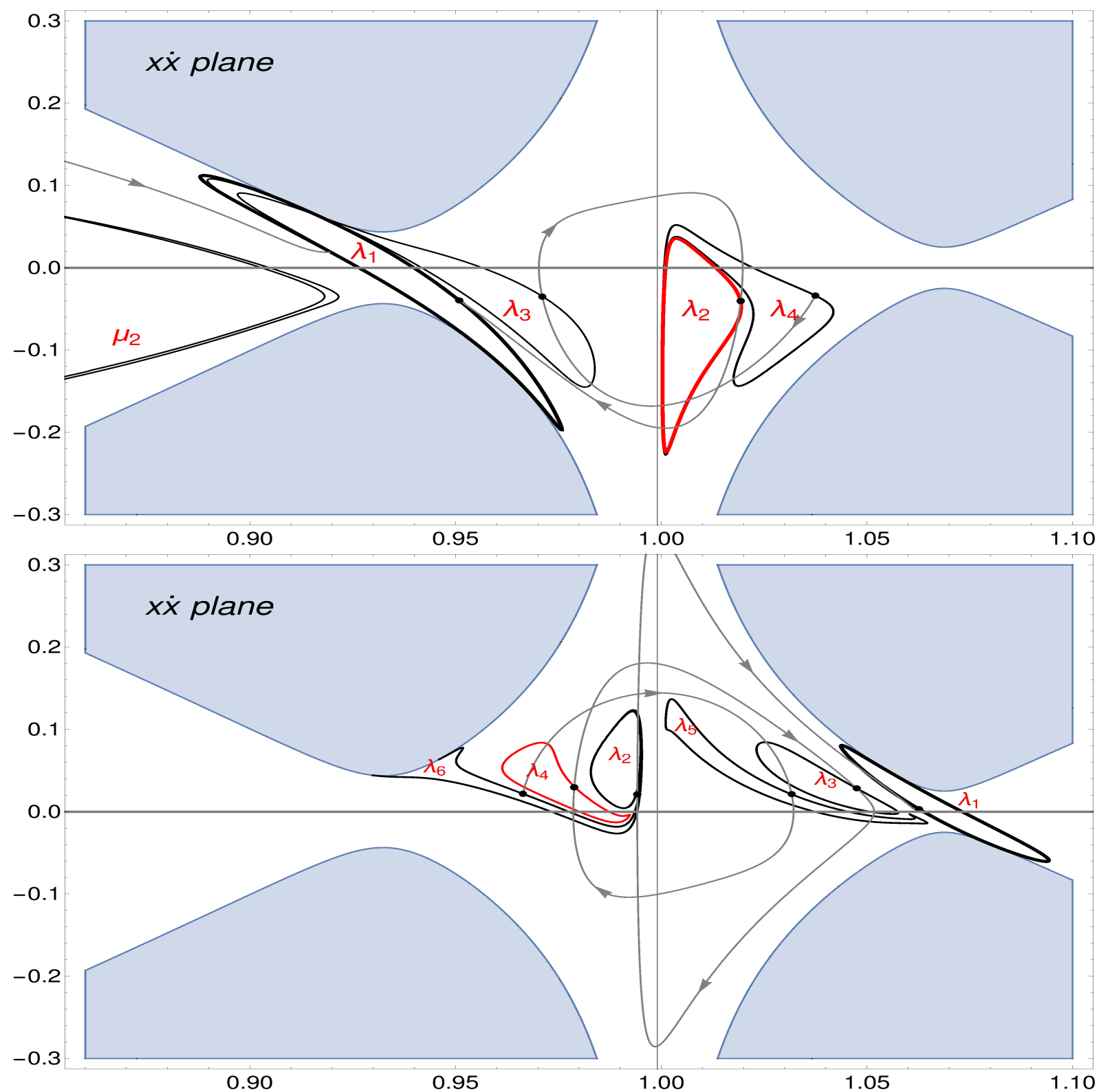

Figure 2: Intersection of $W_{C, 1}^{s}, W_{C, 2}^{s}$ with the planar section $\Sigma_{P}$. We represent the ridges of the mFLI on the vertical section $\Sigma_{P}$, parameterized by $x, \dot{x}$. The modulus $|\dot{y}|$ is obtained from the value of the Jacobi constant. In the top panel we represent the ridges $\mu_{1}^{1}, \lambda_{1}^{1}, \ldots, \lambda_{4}^{1}$ (the superscript is omitted in the figure) which approximate $W_{C, 1}^{s} \cap \Sigma_{P}$. The curves $\lambda_{2}^{1}, \lambda_{4}^{2}$ are ridges of $F L I_{\phi}^{+}$, while the other curves are ridges of $F L I_{\phi}^{-}$; all the points of $\mu_{2}^{1}, \lambda_{2}^{1}, \lambda_{4}^{1}$ are characterized by $\dot{y}>0$, while $\lambda_{1}^{1}, \lambda_{3}^{1}$ contain points with $\dot{y} \geq 0$ and $\dot{y}<0$. In the bottom panel we represent the ridges $\lambda_{1}^{2}, \ldots, \lambda_{6}^{2}$ (the superscript is omitted in the figure) which approximate $W_{C, 2}^{s} \cap \Sigma_{P}$. The curves $\lambda_{1}^{2}, \lambda_{3}^{2}, \lambda_{5}^{2}$ are the ridges of $F L I_{\phi}^{+}$, while the other curves are ridges of $F L I_{\phi}^{-}$; all the points of $\lambda_{2}^{2} \lambda_{5}^{2}$ are characterized by $\dot{y}>0, \lambda_{2}^{2}, \lambda_{4}^{2}, \lambda_{6}^{2}$ by $\dot{y}<0$, while $\lambda_{1}^{1}$ contains points with $\dot{y} \geq 0$ and $\dot{y}<0$. The gray curves represent the projection on $\Sigma_{P}$ of two orbits with initial conditions on $W_{C, 1}^{s}, W_{C, 2}^{s}$ respectively; the orbits return to the section several times, intersecting different connected ridges on the points marked with a black bullet. 
another part performs a circulation around $P_{2}$ intersecting $\Sigma_{V}$ in a point of $\lambda_{3}^{1}$ and a point of $\lambda_{4}^{1}$. In particular, there are orbits tangent to the section $\Sigma_{P}$, determining such bifurcations in the transit properties. The possible transitions are summarized in figure 4, top panel; by extending the computations of the ridges to longer integration times we would detect additional ridges $\lambda_{i}^{1}$ and additional transitions. The topology of the manifold $W_{C, 1}^{s}$ in the realm $S$ (described in [24]) develops like a strip which folds in the interior realm $S$ filling a large portion of $S$.

In the bottom panel of figure 2 we report the ridges of the mFLI on the section $\Sigma_{P}$, computed using a weight function $\phi(\xi)$ which is different from zero only in a small neighbourhood of $L L_{2}$; in the realm $J$ we find six ridges, which we denote by $\lambda_{1}^{2}, \ldots, \lambda_{6}^{2}$. The ridge $\lambda_{1}^{2}$ intersects $L L_{2}$ and extends on both sides of the Lyapunov orbit; all the orbits with initial conditions on $\lambda_{1}^{2}$ converge directly to $L L_{2}$ without performing circulations around $P_{1}, P_{2}$. The tube of orbits generated by the forward-time flow of the three-body problem with initial conditions on $\lambda_{4}^{2}$ (the red curve in the bottom panel of 2) performs a full circulation of $P_{2}$, and intersects the section $\Sigma_{P}$ at $\lambda_{3}^{2}, \lambda_{2}^{2}$ (see figure 5, top panel); then, the orbits with initial conditions on $\lambda_{2}^{2}$ perform an additional half circulation around $P_{2}$ and then intersect the section $\Sigma_{P}$ at some point of $\lambda_{1}^{2}$; afterwards, as all the orbits with initial conditions on $\lambda_{1}^{2}$, they converge directly to $L L_{2}$. Then, we consider the tube of orbits generated by the backward-time flow with initial conditions on $\lambda_{4}^{2}$. Again, the tube perform half a circulation around $P_{2}$ till it intersects $\Sigma_{P}$ in $\lambda_{5}^{2}$; then by continuing the backward integration of orbits with initial conditions on $\lambda_{5}^{2}$ we have three different possibilities: the orbit intersect $\Sigma_{P}$ at $\lambda_{6}^{2}$, or they transit to the external realm $E$, or they transit to the internal realm $S$. The possible transitions are summarized in figure 4 , bottom panel.

\subsection{The vertical section of the hypertube manifolds}

In the figures 6 and 7 we report the ridges of the mFLI on the section $\Sigma_{V}$, computed using both the weight functions $\phi_{1}(\xi), \phi_{2}(\xi)$ defined in Section 5. For $x>1-\mu$ we report the ridges of the $F L I_{\phi_{i}}^{+}$indicator, while for $x<1-\mu$ we report the ridges of the $F L I_{\phi_{i}}^{-}$indicator: since all the ridges do not cross the line $x=1-\mu$, we are allowed to use the two modified FLI in place of the more CPU expensive modified FTLE.

The black curves are ridges of $F L I_{\phi_{1}}^{ \pm}$: the bold black curve, which we denote by $\Lambda_{1}^{1}$, represents the intersection of the center manifold $W_{C, 1}^{c}$ with the vertical section $\Sigma_{V}$; the other ridges represent intersections $W_{C, 1}^{s} \cap \Sigma_{V}$. Two of these curves, which we denote by $M_{1}^{1}, M_{2}^{1}$, belong to the left hand side branch of the stable manifold (see figure 7 ) and are in the realm $S$ : the initial conditions on $M_{1}^{1}$ (which is on the right of the primary body) perform half of a circulation around the primary body before approaching the center manifold; the initial conditions on $M_{2}^{1}$ (which is on the left of the primary body) perform a complete circulation around the primary body before approaching the center manifold. The remaining curves (light black curves in figure 6) are on 

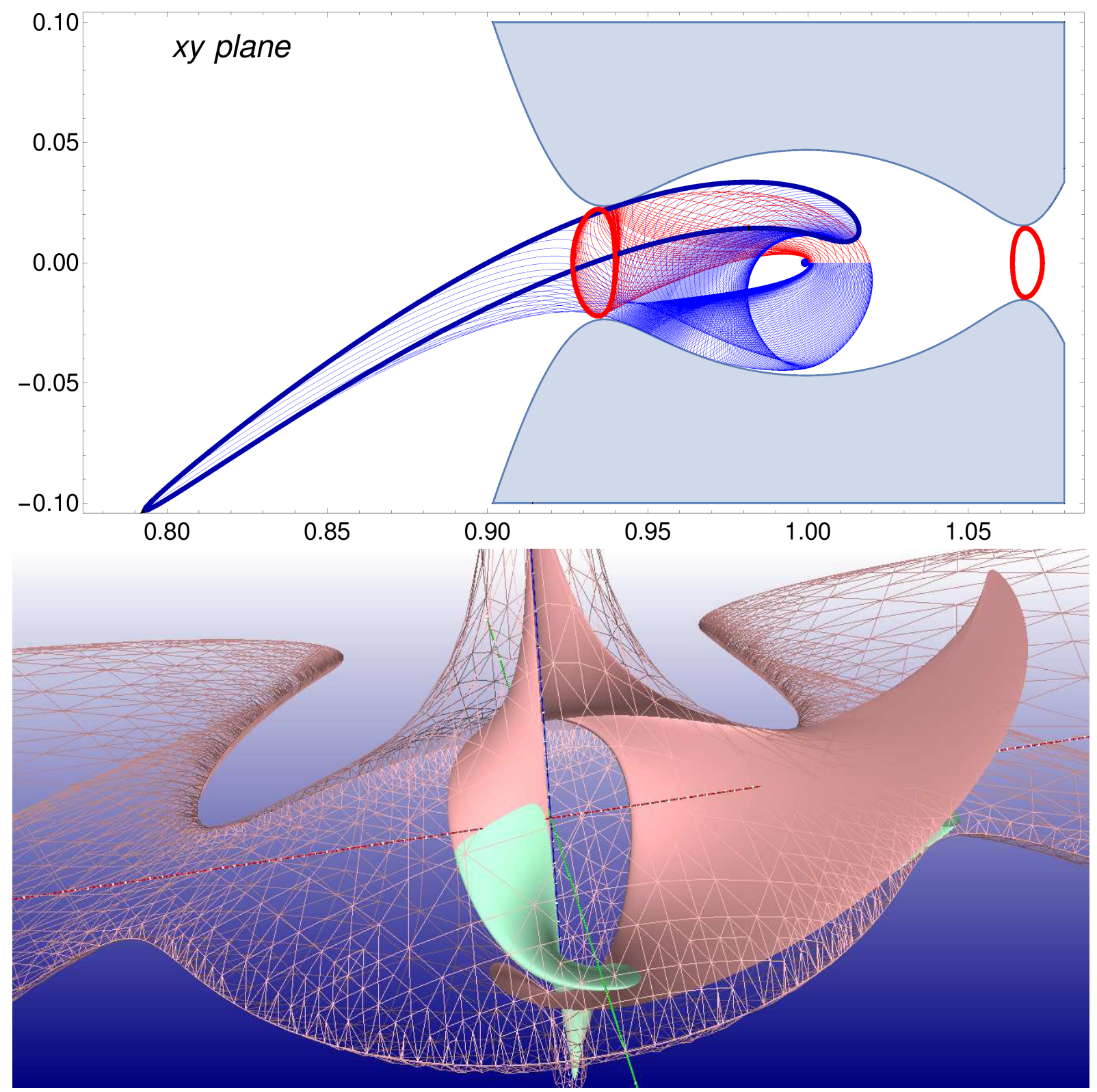

Figure 3: Tube of orbits generated by $\lambda_{2}^{1}$ obtained by integrating a sample of initial conditions both for positive and negative times. On the top panel we represent the projection of these orbits on the $x, y$ plane. The red curves represent the orbits integrated for positive times; the blue curves represent the orbits integrated up to the negative proper time $s=-45$. The bold blue curve represents the border of the tube at $s=-45$. In the bottom panel we represent the tubes generated by the same orbits in the three-dimensional phase-space $x, y, \dot{x}$; a transparent mesh samples the border of $\mathcal{M}_{C}$. The light-green surface is the portion of stable-manifold obtained by integrating forward the initial conditions on $\lambda_{2}^{1}$, the pink surface represents the tube obtained by integrating backward the initial conditions on $\lambda_{2}$. The perspective is reversed with respect to the upper patrel: the positive $x$ axis points toward the left. 

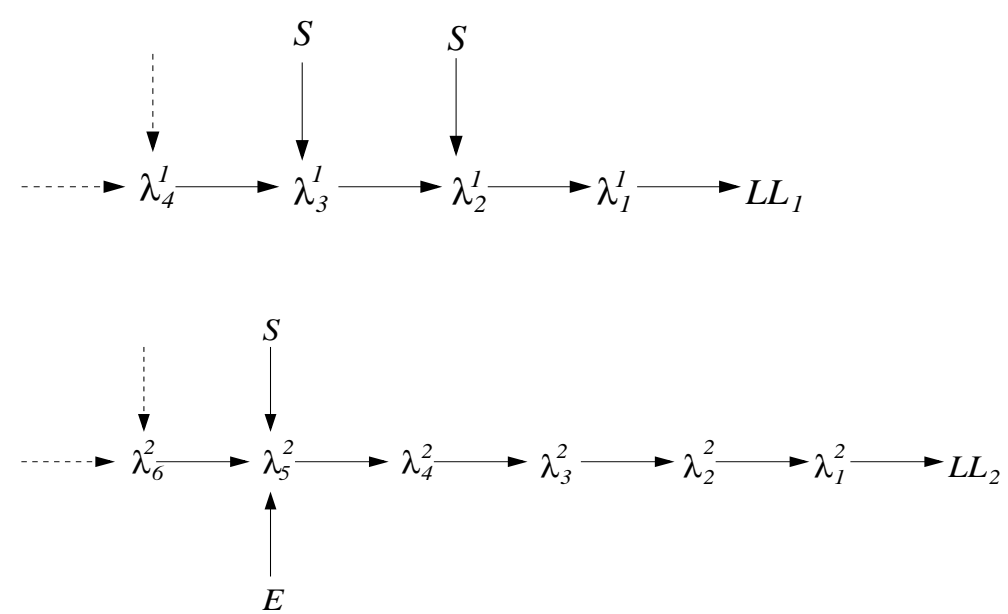

Figure 4: Transitions between the curves $\lambda_{j}^{i}$ and the realms $\mathbf{J}$ and $\mathbf{S}$. In the top panel we summarize the transition properties for the orbits with initial conditions on $\lambda_{i}^{1}$. The forward time evolution is simple: any initial conditions in $\lambda_{i}^{1}$ will intersect the section in $\lambda_{i-1}^{1}, \ldots, \lambda_{1}^{1}$ and then converges to $L L_{1}$. The backward time evolution has bifurcations, summarized by the diagram. In the bottom panel we summarize the transition properties for the orbits with initial conditions on $\lambda_{i}^{2}$. The forward time evolution is simple: any initial conditions in $\lambda_{i}^{2}$ will intersect the section in $\lambda_{i-1}^{2}, \ldots, \lambda_{1}^{2}$ and then converges to $L L_{2}$. The backward time evolution has bifurcations, summarized by the diagram.

the right hand-side branch of the stable manifold, and we denote them by $\Lambda_{2}^{1}, \Lambda_{3}^{1}, \ldots, \Lambda_{7}^{1}$; where the lower index $j$ labels the number $(j-1) / 2$ of circulations around the secondary body before entering permanently the region $R_{1}$. These ridges intersect the corresponding planar ridges $\lambda_{j}^{1}$ (reported in figure 2, top panel) at the one dimensional section:

$$
\Sigma_{V P}=\{(x, y, z, \dot{x}, \dot{y}, \dot{z}): y=z=0, \dot{x}=\dot{z}=0, \mathcal{C}(x, 0,0, \dot{y}, 0)=C\} .
$$

As a matter of fact, we found two disconnected ridges $\Lambda_{4}^{1}, \tilde{\Lambda}_{4}^{1}$ such that the orbits with initial conditions on both of them perform $3 / 2$ circulations around $P_{2}$ before entering permanently the region $R_{1}$, see the bottom panels of figure 6 . These two curves are disconnected in $\Sigma_{V}$ but are connected in the phase-space $\mathcal{M}_{C}$ through the intersection $W_{C, 1}^{s} \cap \Sigma_{P}$ of the stable manifold with the planar section, see figure 8. Such connections are expected to exist also for other ridges, and are important to determine the transit properties of the orbits with initial conditions on $\Sigma_{V}$, see Subsection 3.3.

The blue curves are ridges of $F L I_{\phi_{2}}^{ \pm}$: the bold blue curve, which we denote by $\Lambda_{1}^{2}$, represents the intersection of the center manifold $W_{C, 2}^{c}$ with the vertical section $\Sigma_{V}$, while the other ridges of $F L I_{\phi_{2}}^{ \pm}$(light blue curves in figure 6) are in the right left-hand side branch of the stable manifold $W_{C, 2}^{s} \cap \Sigma_{V}$. We denote them by $\Lambda_{3}^{2}, \ldots, \lambda_{7}^{2}$, where the lower index $j$ labels the number $(j-1) / 2$ of circulations around the secondary body before entering permanently $R_{2}$ (see figure 


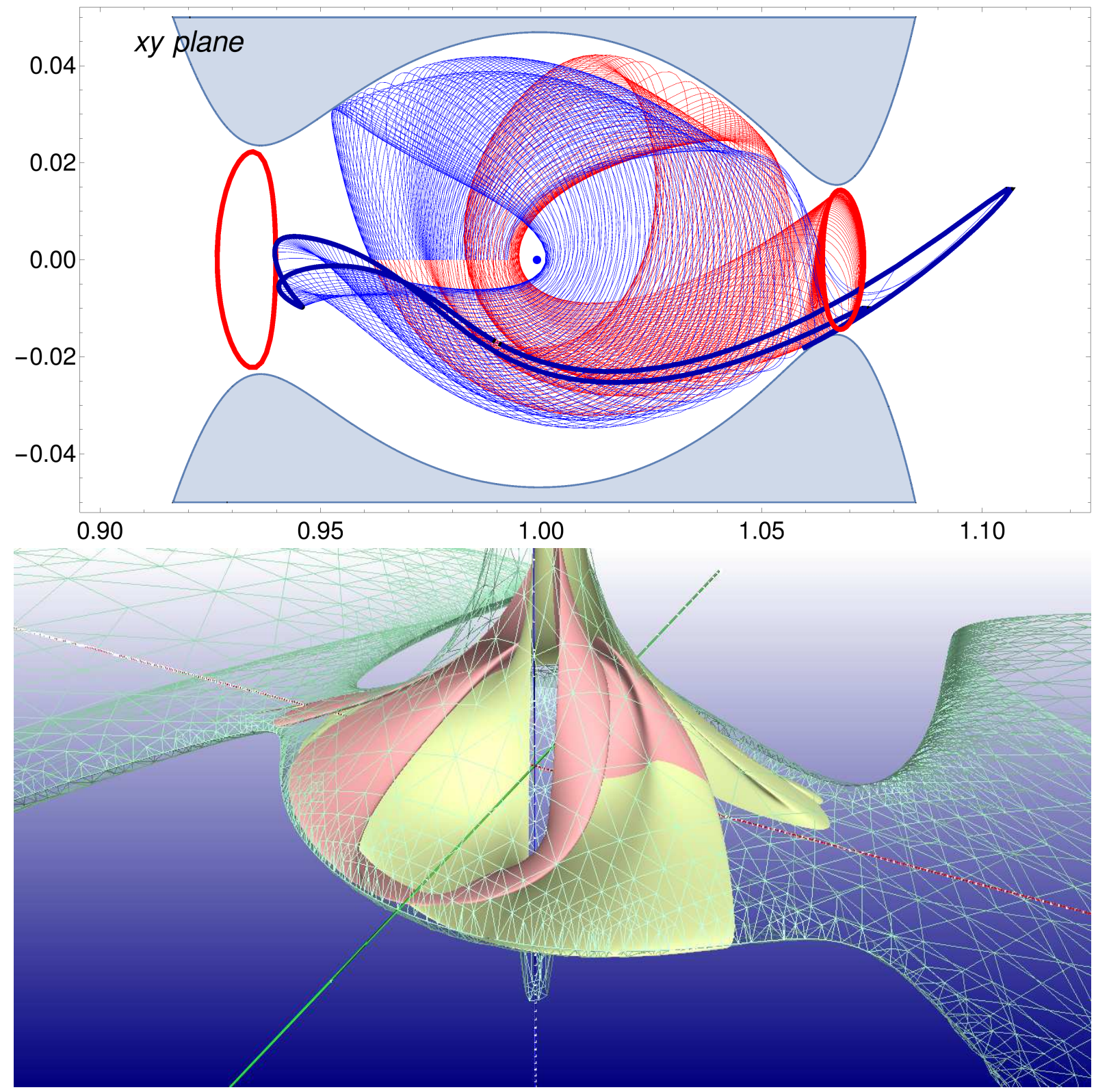

Figure 5: Tube of orbits generated by $\lambda_{4}^{2}$ obtained by integrating a sample of initial conditions both for positive and negative times. On the top panel we represent the projection of these orbits on the $x, y$ plane. The red curves represent the orbits integrated for positive times; the blue curves represent the orbits integrated for negative times. The bold blue curve represents the border of the tube. In the bottom panel we represent the tubes generated by the same orbits in the three-dimensional phase-space $x, y, \dot{x}$; a transparent mesh samples the border of $\mathcal{M}_{C}$. The light-green surface is the portion of stablemanifold obtained by integrating backward the initial conditions on $\lambda_{4}^{2}$, the pink surface represents the tube obtained by integrating forward the initial conditions on $\lambda_{4}^{2}$. The perspective is reversed with respect to the upper panel: the positive $x$ axis points towart the left. 
1). These ridges intersect at $\Sigma_{V P}$ the corresponding planar ridge $\lambda_{j}^{2}$ reported in figure 2 , bottom panel.

Methods of grids computation. The ridges represented in this paper are computed as an ordered sequence of close points $\pi_{0}, \pi_{1}, \ldots$. Since the sections $\Sigma_{V}$ and $\Sigma_{P}$ intersect at the one dimensional section $\Sigma_{V P}$, we preliminary compute the modified FLI for a grid of initial conditions on $\Sigma_{V P}$. Each maximum of the FLI on this one dimensional grid is close to a ridge of the FLI. Precisely, we select a point $\tilde{\pi}_{0}$ corresponding to one of these maxima, and we compute again the modified FLI on a much more refined one-dimensional grid centered in $\tilde{\pi}_{0}$. The maximum FLI on this new grid provides a better estimate of the the first point $\pi_{0}$ on the ridge of the FLI. The second point $\pi_{1}$ of the ridge is obtained by computing the FLI on another one-dimensional grid of points on $\Sigma_{V}$ characterized by a small value $\varepsilon_{0}$ of the coordinate $z$, and the same coordinates $x, y, \dot{x}, \dot{z}$ of $\pi_{0}$; the value of $|\dot{y}|$ is determined from the value of the Jacobi constant. The maximum FLI on this second grid provides $\pi_{1}$. The third point $\pi_{2}$ is computed from a one-dimensional grid of $N$ points chosen on an segment which is orthogonal to $\pi_{1}-\pi_{0}$, whose center is at a distance $\varepsilon_{0}$ from $\pi_{1}$, and whose amplitude is equal to another small parameter $\varepsilon_{1}$. The maximum FLI on this grid provides the point $\pi_{2}$. The procedure is then iterated, providing an ordered set of points on a curve which samples a ridge of the mFLI with maximum error $\varepsilon_{1} / N$. The values of the parameters $\varepsilon_{0}, \varepsilon_{1}, N$ are adjusted at each step. Then, we select a subset of points $\pi_{i}$, and we refine the computation by recomputing the FLI on one dimensional grids centered on $\pi_{i}$, orthogonal to $\pi_{i}-\pi_{i-1}$, and with a new value for $\varepsilon_{1}$ which is set equal to $\varepsilon_{1} / 2$. After $K$ of these refinements, we obtain an ordered set of points on a curve which samples a ridge of the mFLI with maximum error $\varepsilon_{1} /\left(2^{K} N\right)$. After few iterations $K$ of this process we arrive a the limit of the double precision floating point arithmetics (to reach the double precision in the computation of the manifold we perform the last steps of the iteration with the extended precision floating point arithmetics).

The equations of motion (16) and their variational equations (17) are numerically integrated with an explicit Runge-Kutta integration scheme of order six using double or extended floating point precision.

\subsection{Survey of the dynamics in the section $\Sigma_{V}$}

In this section we represent the dynamics of the orbits with initial conditions in the section $\Sigma_{V}$, with particular attention to the orbits with initial conditions close to the ridges of the chaos indicators previously computed.

We first represent the tube of orbits with initial conditions on the ridges $\Lambda_{j}^{i}$ of the modified FLIs. Ideally, these orbits converge to the center manifold $W_{C, i}^{c}$ for both positive and negative times; but, since the ridges approximates the stable/unstable manifolds up to a numerical error, 

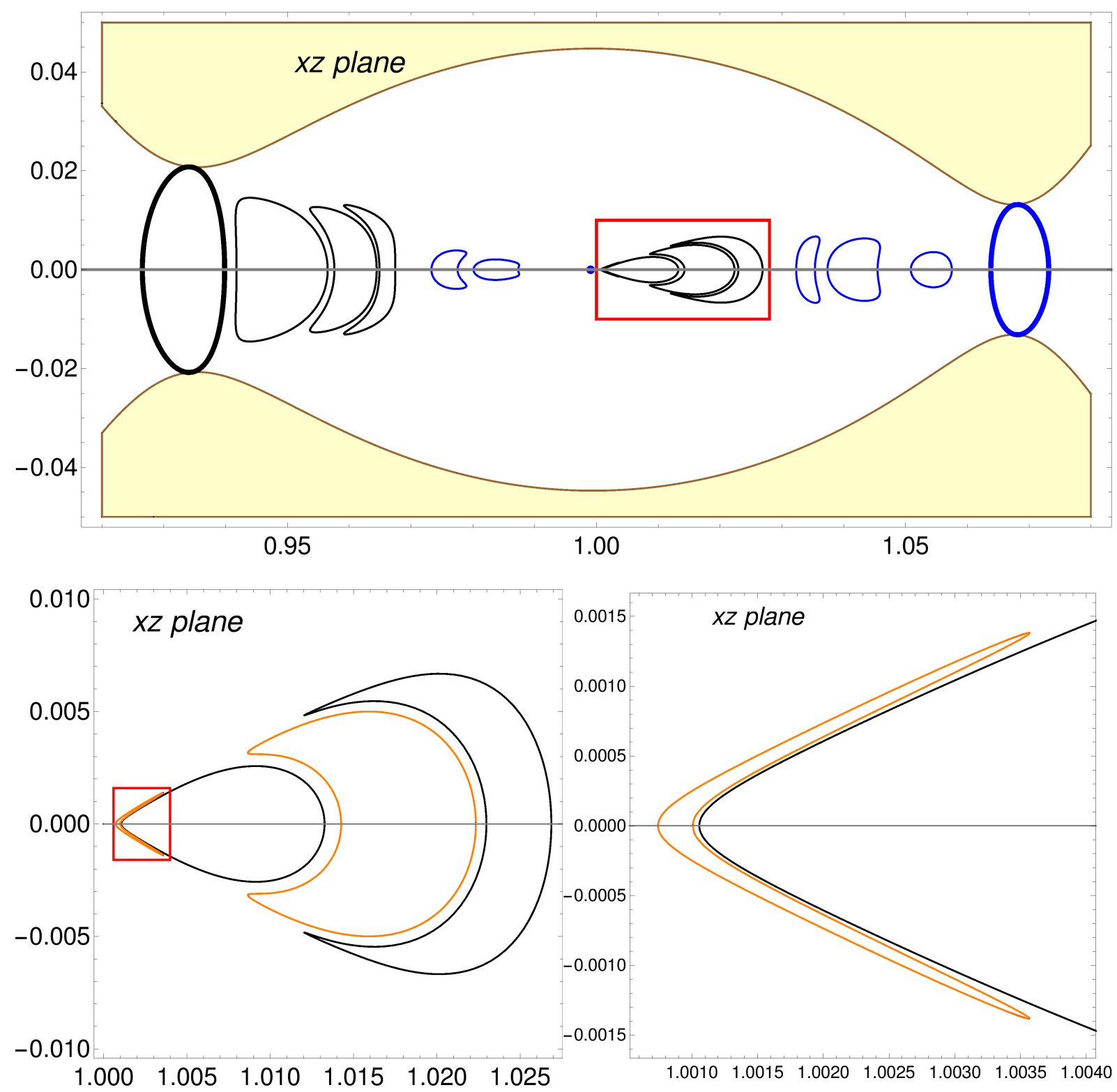

Figure 6: Intersection of $W_{C, 1}^{s}$ with the vertical section $\Sigma_{V}$ in the realm $J$. We represent the ridges of the mFLI on the vertical section $\Sigma_{V}$, parameterized by $x, z$. The modulus $|\dot{y}|$ is obtained from the value of the Jacobi constant; the other initial conditions are $y, \dot{x}, \dot{z}=0$. The black curves have been obtained from $F L I_{\phi_{1}}^{ \pm}$: the bold black curve $\Lambda_{1}^{1}$ represents $W_{C, 1}^{c} \cap \Sigma_{V}$, the light black curves represent the $\Lambda_{2}^{1}, \ldots, \Lambda_{7}^{1}$. The blue curves have been obtained from $F L I_{\phi_{2}}^{ \pm}$: the bold blue curve $\Lambda_{1}^{2}$ represents $W_{C, 2}^{c} \cap \Sigma_{V}$, the light blue curves represent the $\Lambda_{2}^{2}, \ldots, \Lambda_{6}^{2}$. In the bottom panels we zoom in the red boxes. From the zooms, we notice an additional ridge, which is the $\tilde{\Lambda}_{4}^{1}$ discussed in the text, connected to the $\Lambda_{4}^{1}$ through the planar ridge $\lambda_{4}^{1}$. Both ridges $\Lambda_{2 \times} \tilde{\Lambda}_{4}^{1}$ are represented in orange in the bottom panels. 

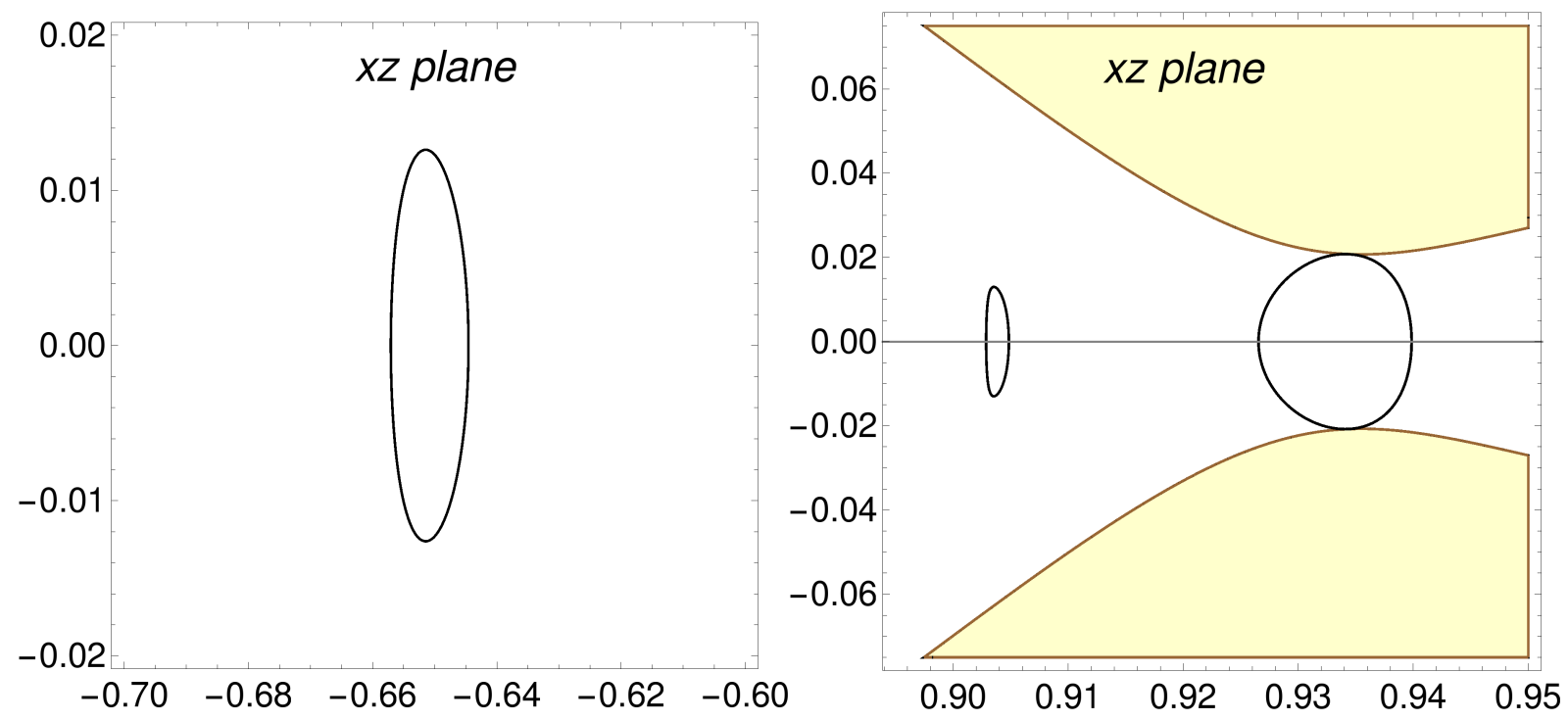

Figure 7: Intersection of $W_{C, 1}^{s}$ with the vertical section $\Sigma_{V}$ in the realm $S$. The light black curves represent the ridges $M_{1}^{1}$ (left panel) and $M_{2}^{1}$ (right panel).

which in this case is $\varepsilon_{1} \sim 10^{-14}$, the orbits approach and remain close to $W_{C, i}^{c}$ only up to some finite time $T$. In figures 9 and 10 we represent the tubes generated by $\Lambda_{2}^{1}$ and $\Lambda_{4}^{1}$ respectively: in the top panels we represent the projection of a sample of orbits on the $x y$ (top-left) and the $x z$ (top-right) planes; the orbits represented in bold-red are on the planar section of the lobes, while the orbits represented in bold-blue are close to the 'cusps' of $\Lambda_{4}^{1}$. We notice the sense of rotation around $P_{2}$ of the orbits, which is anti-clockwise, until they enter the realm $R_{1}$, where they change their rotation sense to follow a clockwise rotation around the center manifold. In the bottom panels we represent the projection of the tubes on the three dimensional space $x y z$.

Even if the numerical error is very small, by extending the integration time these orbits will transit through the bottleneck of $\mathcal{B}_{C}$ at $L_{1}$ or bounce back, depending on which side of the stable manifold they actually are. As a matter of fact, the meaning of the numerical computation of each curve $\Lambda_{j}^{i}$ is the determination of an annulus $\sigma_{j}^{i} \subseteq \Sigma_{V}$ delimited by an inner curve and an outer curve, whose points are at distance from $\Lambda_{j}^{i}$ larger than the numerical error $\varepsilon_{1}$. Therefore, with the exception of the points of the annulus $\sigma_{j}^{i}$, we are able to identify with numerical error $\varepsilon_{1}$ the interior and the exterior of the tubes of orbits generates by $W_{C, i}^{s} \cap \Sigma_{V}$. The dynamics of orbits with initial conditions in the tubes approximated by a $\Lambda_{j}^{i}$ is such that, after $(j-1) / 2$ circulations around $P_{2}$, the orbits transit to the internal realm $S$, if $i=1$, or to the external realm $E$, if $i=2$. In figure 11 we report the different time evolutions of the internal and external 

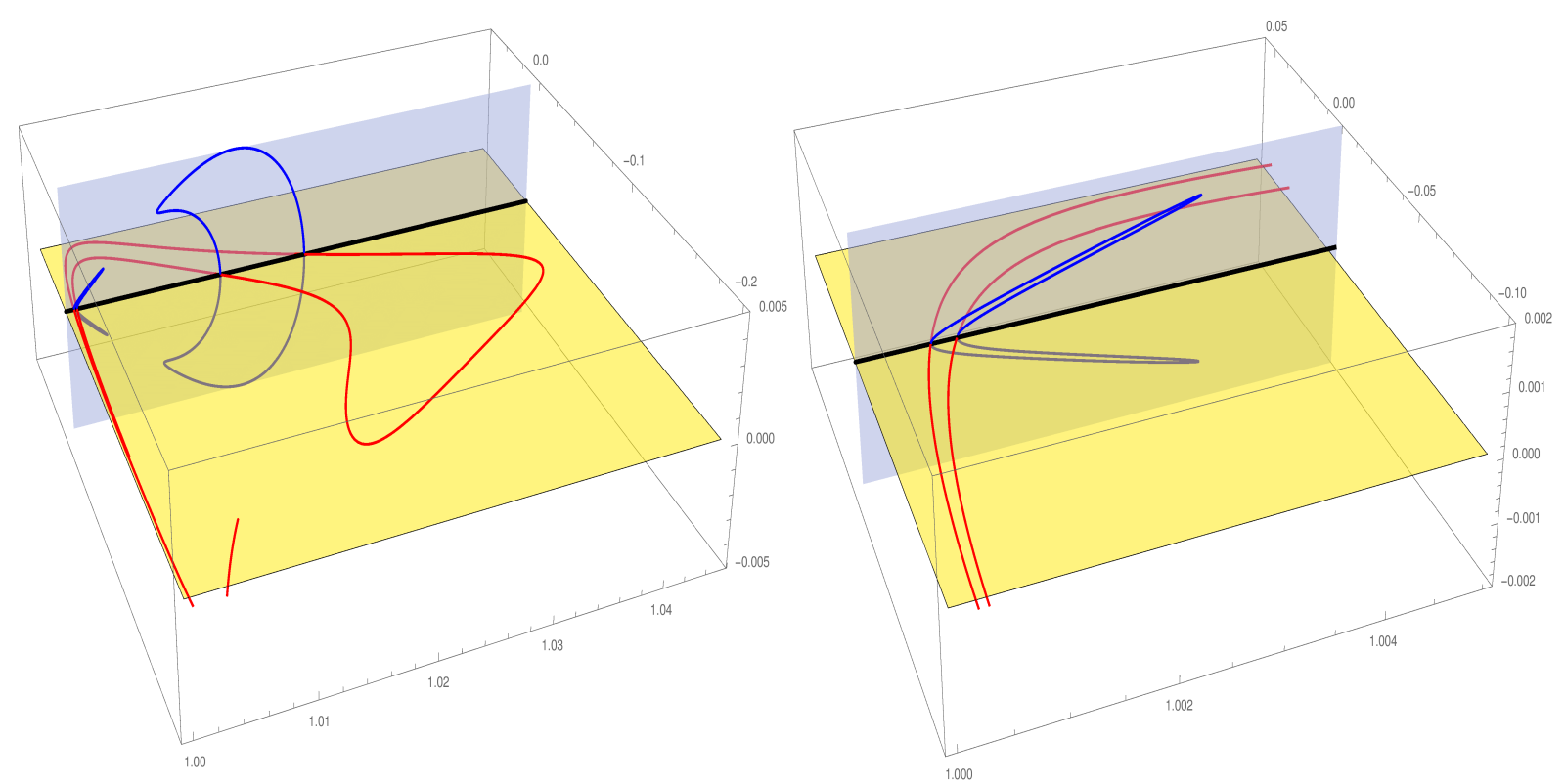

Figure 8: Intersection of the ridges $\Lambda_{4}^{2}, \tilde{\Lambda}_{4}^{2}$ with $\lambda_{4}^{2}$. The ridges $\Lambda_{4}^{2}, \tilde{\Lambda}_{4}^{2}$ of $\Sigma_{V}$ and the ridge $\lambda_{4}^{2}$ of $\Sigma_{P}$ are all represented in the same three-dimensional section of the phase-space $\Sigma=\{(x, y, z, \dot{x}, \dot{y}, \dot{z})$ : $y=0, \dot{z}=0, \mathcal{C}(x, y, z, \dot{x}, \dot{y}, \dot{z})=C\}$. The section is parametrized by the variables $x, z, \dot{x}$, so that the horizontal yellow plane represents the planar section $\Sigma_{P}$, the blue vertical plane represents the vertical section $\Sigma_{V}$. The ridges $\Lambda_{4}^{2}, \tilde{\Lambda}_{4}^{2}$ are clearly connected by $\lambda_{4}^{2}$.

borders of the two annuli $\sigma_{2}^{1}, \sigma_{4}^{2}$ (whose borders have distance $\varepsilon=10^{-10}$ from $\lambda_{j}^{i}$; we selected $\varepsilon$ much larger than the numerical precision, to appreciate the dynamics of all the orbits on the border in short times): for $i=1,2$ all the orbits in the inner border, after some circulations of the center manifold, transit to $S, E$ respectively; all the orbits in the external border, after some circulations of the center manifold, bounce back to the realm $J$.

The transit properties of orbits which are external to the tubes is in part uncertain, since there can be different branches of $\Lambda_{j}^{i}$ (see figure 8) which are disconnected in $\Sigma_{V}$ but are connected through $\Sigma_{P}$ : an initial condition which is external to a connected component of $\Lambda_{j}^{i}$, but internal to a different connected component of $\Lambda_{j}^{i}$, transits to the realm $S$ in $(j-1) / 2$ circulations. Therefore, while the dynamics of the inner border of the $\sigma_{j}^{i}$ is certainly determined, the dynamics on the external border of $\sigma_{j}^{i}$ is determined for a certain integration time $T$ only if the error $\varepsilon_{1}$ is suitably small. Global snapshots of the transit properties from the realm $J$ to the realms $S, E$ are provided in figure 12. For any initial condition on a refined grid of $10000 \times 2000$ initial conditions of $\Sigma_{V}$ we computed their orbits until they reach a value for the $x$ coordinate smaller than 0.933 or larger than 1.067. In the former case, we represent the initial condition with a violet pixel, in the latter we represent the initial condition with an orange pixel. 
If none of the conditions is met in a certain fixed integration time $T$, we represent the initial condition with a yellow pixel. Though such representations have a poor precision with respect to the FLIs computations (here, the precision in separating the interior from the exterior of a tube is provided by the step-size of the grid, and is 9 orders of magnitude worst than the FLI computations), they provide a snapshot of the global dynamics on the section $\Sigma_{V}$.

\section{Variational equations and regularizations}

In this Section we discuss the properties of the variational equations of the restricted three-body problem defined from the KS variables. First, we review the KS transformation regularizing the equations of motions of the three-body problem; then we discuss the invariance properties of the solutions of the variational equations of the regularized equations of motion, and compare them with the solutions of the variational equations of the non-regularized equations of motion.

\subsection{Invariance properties of the KS transformation}

The inverse of the KS transformation (6), and of its phase-space extension (14), are undetermined: in fact the projection $\chi(u, \nu)$ of any $(u, \nu) \in T \mathbb{U}_{0}$ on the Cartesian variables $(x, y, z, \dot{x}, \dot{y}, \dot{z})$ is invariant under the transformation:

$$
u \mapsto \mathcal{R}_{\alpha} u \quad, \quad \nu \mapsto \mathcal{R}_{\alpha} \nu
$$

where:

$$
\mathcal{R}_{\alpha}=\left(\begin{array}{cccc}
\cos \alpha & 0 & 0 & -\sin \alpha \\
0 & \cos \alpha & \sin \alpha & 0 \\
0 & -\sin \alpha & \cos \alpha & 0 \\
\sin \alpha & 0 & 0 & \cos \alpha
\end{array}\right)
$$

for all $\alpha \in[0,2 \pi]$. In particular, the pre-image of the Lagrangian points $L_{1}, L_{2}$ defined by the map $\chi$ is the set of the $(u, \nu):=\left(u^{*}(\alpha), 0\right)$ with:

$$
\begin{aligned}
& u_{1}^{*}(\alpha)=u_{4}^{*}(\alpha)=0, u_{2}^{*}(\alpha)=\sqrt{\gamma_{1}} \cos \alpha, u_{3}^{*}(\alpha)=\sqrt{\gamma_{1}} \sin \alpha\left(\text { for } L_{1}\right) \\
& u_{2}^{*}(\alpha)=u_{3}^{*}(\alpha)=0, u_{1}^{*}(\alpha)=\sqrt{\gamma_{2}} \cos \alpha, u_{4}^{*}(\alpha)=\sqrt{\gamma_{2}} \sin \alpha\left(\text { for } L_{2}\right)
\end{aligned}
$$

where $\alpha \in[0,2 \pi]$, and $\gamma_{1}, \gamma_{2}$ denote the Cartesian distances of the equilibria configurations $L_{1}, L_{2}$ from $P_{2}$.

Then, we consider the extension of the projection (14) to the map:

$$
\tilde{\chi}: T \mathbb{U}_{0} \longrightarrow \mathbb{R}^{8}
$$



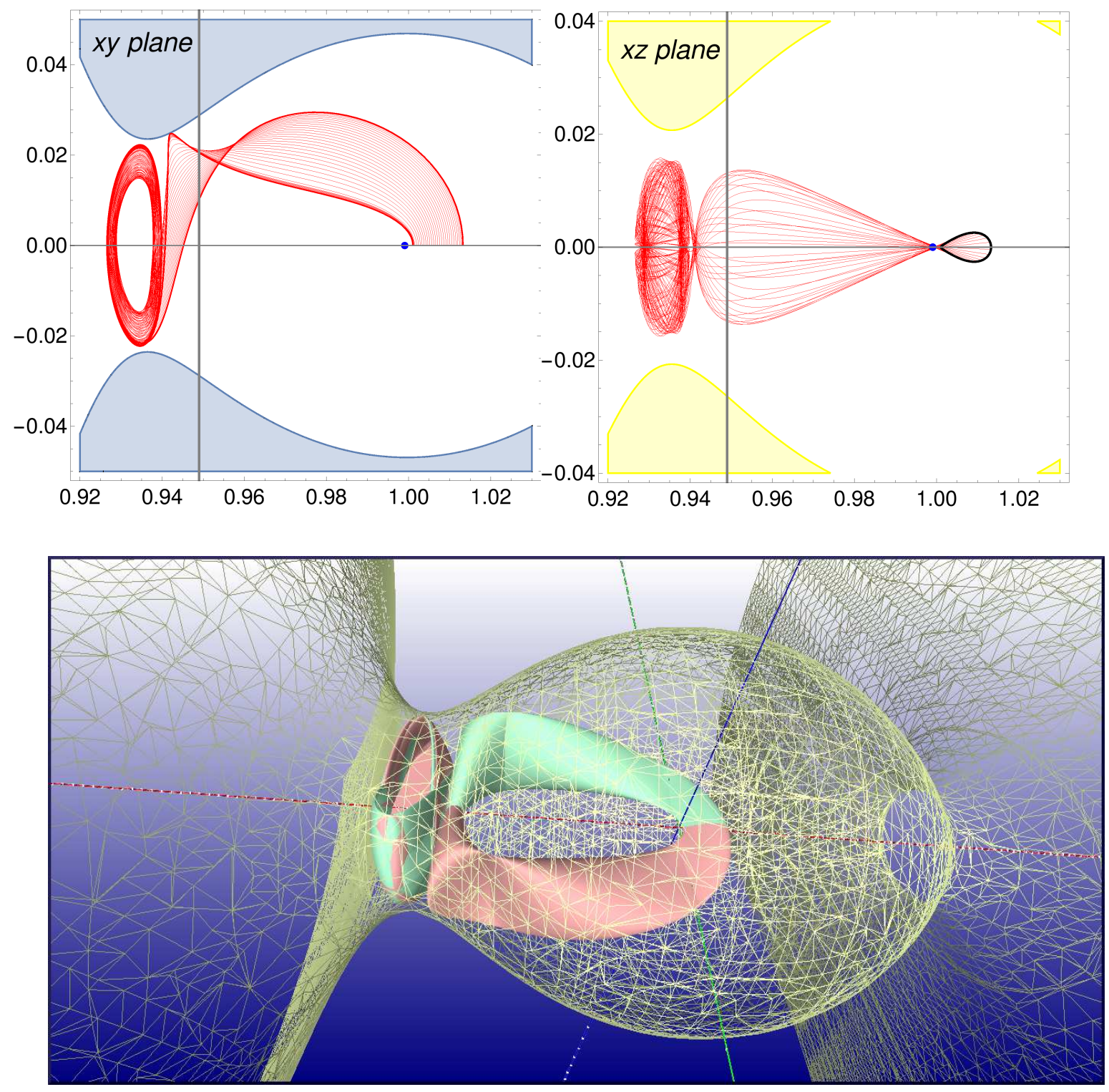

Figure 9: Geometry of the tube generated by $\Lambda_{2}^{1}$. A sample of orbits in $\Lambda_{2}^{1}$ has been integrated forward in time. The top-left panel represents the projection of these orbits on the $x y$ plane (the the two orbits represented in bold are on the planar section of the lobe); the top-right panel represents the projection of these orbits on the $x z$ plane. The light-blue and the yellow regions represent the forbidden regions in the plane $x y$ and $x z$ respectively. Then, the same initial conditions have been integrated backward in time. In the bottom panel we represent in the three-dimensional space $x y z$ the mesh generated by the orbits obtained with the forward integration (represented in green) and with the backward integration (represented in pink). The transparent mesh represents the border of the real of the forbidden motions. The pink disk inside the center manifold is a surface whose border is the Lyapunov orbit $L L_{1}$. 

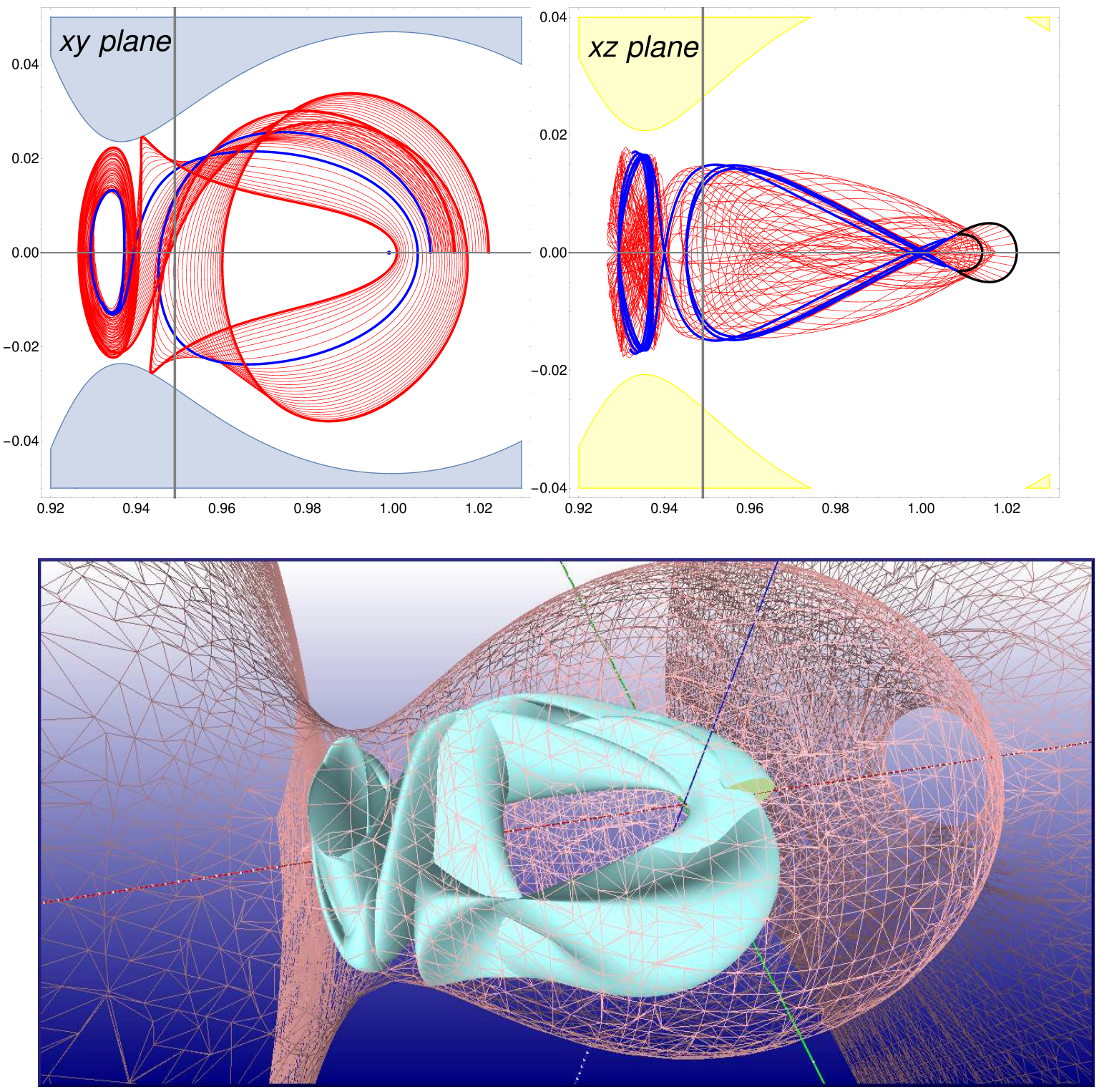

Figure 10: Geometry of the tube generated by $\Lambda_{4}^{1}$. A sample of orbits in $\Lambda_{4}^{1}$ has been integrated forward in time. The top-left panel represents the projection of these orbits on the $x y$ plane, the top-right panel represents the projection of these orbits on the $x z$ plane (the two orbits represented in red-bold are on the planar section of the lobe; the two orbits represented in blue have initial conditions close to the 'cusps' of $\lambda_{4}$ ). The light-blue and the yellow regions represent the forbidden regions in the plane $x y$ and $x z$ respectively. In the bottom panel we represent in the three dimensional space $x y z$ the mesh generated by the same orbits (represented in light blue). The transparent mesh represents the border of the forbidden region. 

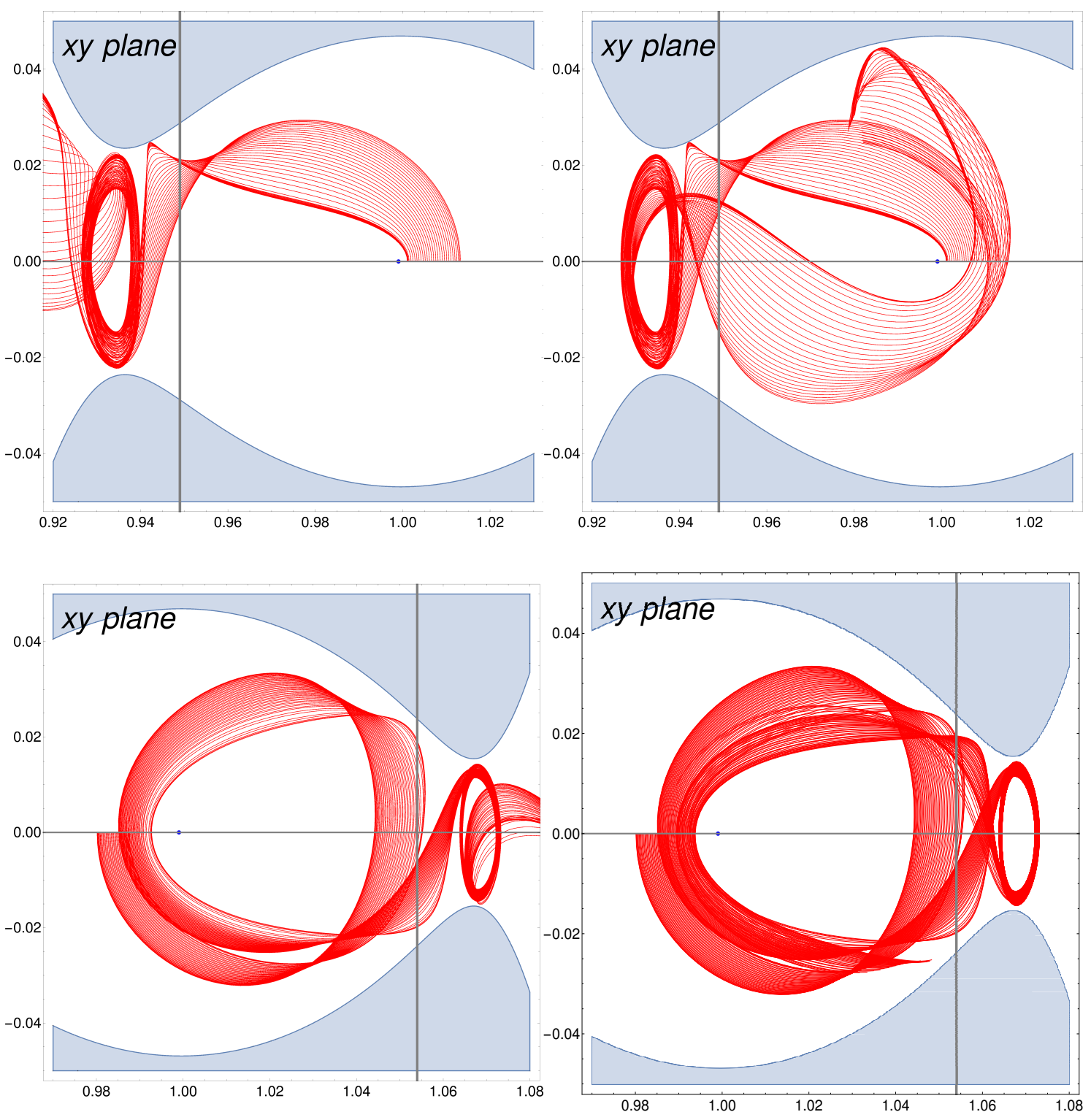

Figure 11: Evolution of orbits with initial conditions on the annuli $\sigma_{2}^{1}, \sigma_{4}^{2}$. We represent the projection on the $x y$ plane of orbits with initial conditions in a sample of the inner border (top-left panel) and outer border (top-right panel) of $\sigma_{2}^{1}$, and on the inner border (bottom-left panel) and outer border (bottom-right panel) of $\sigma_{4}^{2}$. 

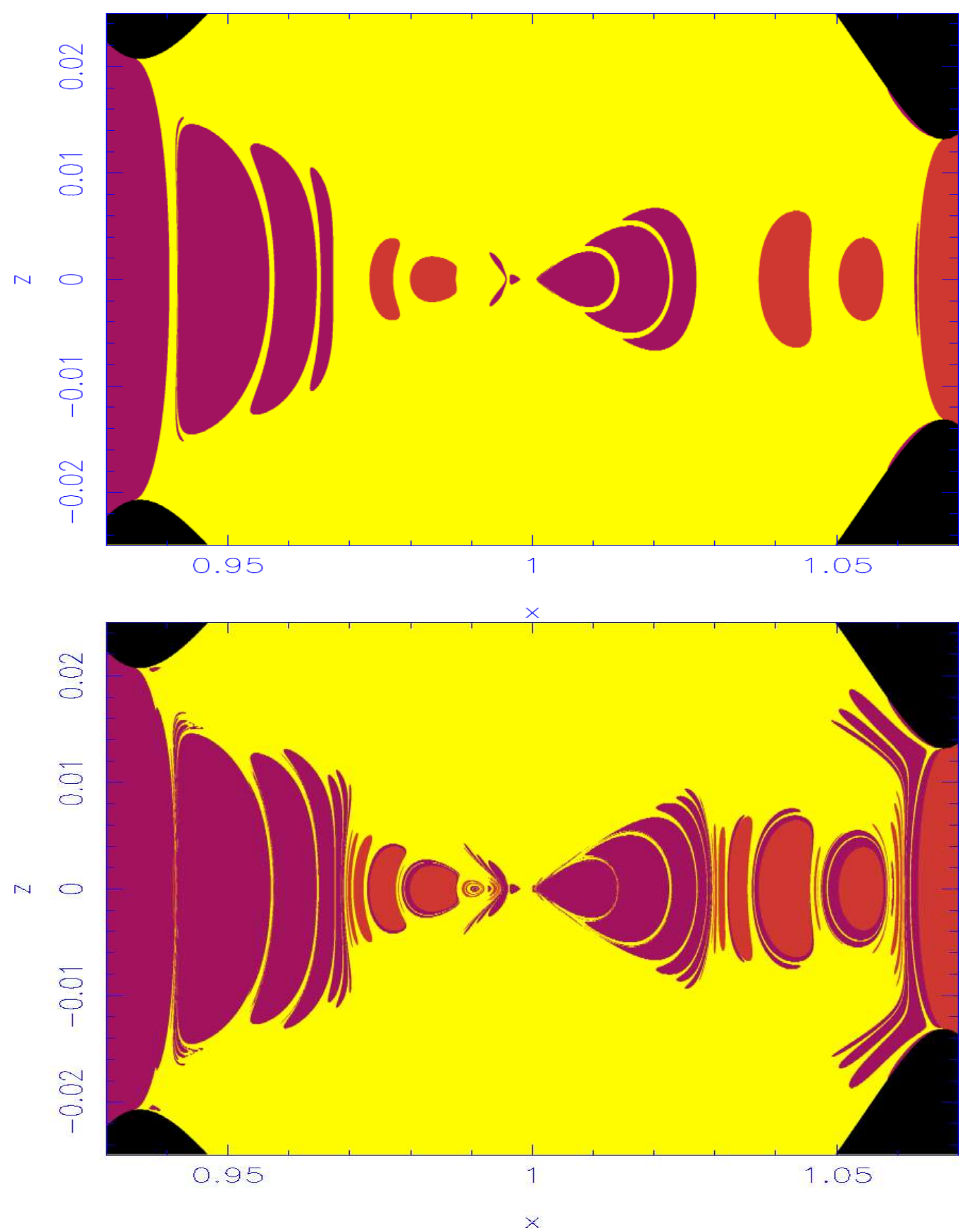

Figure 12: Transits from the realm $J$ to the realms $S, E$. For any initial condition on a refined grid of $10000 \times 2000$ initial conditions of $\Sigma_{V}$ we computed their orbits until they reach a value for the $x$ coordinate smaller than 0.933 or larger than 1.067. In the former case, we represent the initial condition with a violet pixel, in the latter we represent the 333tial condition with an orange pixel. If none of the conditions is met in a certain fixed integration time $T$, we represent the initial condition with a yellow pixel. The top panel has been obtained with the integration time $T=5$; the bottom panel with the integration time $T=10$. 


$$
\left(u_{1}, u_{2}, u_{3}, u_{4}, \nu_{1}, \nu_{2}, \nu_{3}, \nu_{4}\right) \longmapsto\left(x, y, z, q, v_{x}, v_{y}, v_{z}, v_{q}\right)
$$

with:

$$
\begin{gathered}
(x, y, z, q)=A(u) u \\
\left(v_{x}, v_{y}, v_{z}, v_{q}\right)=\frac{1}{\|u\|^{2}}(A(u) \nu+A(\nu) u)=\frac{2}{\|u\|^{2}} A(u) \nu
\end{gathered}
$$

where the last equality in the second line of (42) is valid for $u \cdot \Omega \nu=0$.

The tangent map of the transformation (41) defines a projection from the space of vectors tangent to $T \mathbb{U}_{0}$ to the space of vectors tangent to $T \mathbb{R}^{8}$. Precisely, for any $(u, \nu) \in T \mathbb{U}_{0}$ with $u \cdot \Omega \nu=0$, consider the map:

$$
\begin{aligned}
D \tilde{\chi}_{(u, \nu)}: T_{(u, \nu)} T \mathbb{U}_{0} & \longrightarrow T_{\tilde{\chi}(u, \nu)} \mathbb{R}^{8} \\
w:=\left(w_{u}, w_{\nu}\right) & \longmapsto V=\left(V_{r}, V_{v}\right)
\end{aligned}
$$

defined by:

$$
\left(\begin{array}{c}
V_{r} \\
V_{v}
\end{array}\right)=\left(\begin{array}{l}
A(u) w_{u}+A\left(w_{u}\right) u \\
\frac{2}{\|u\|^{2}}\left(A\left(w_{u}\right) \nu+A(u) w_{\nu}-\frac{2}{\|u\|^{2}} u \cdot w_{u} A(u) \nu\right)
\end{array}\right) .
$$

Because of the non-holonomic constraint $u \cdot \Omega \nu=0$, we restrict the tangent map (44) to the set:

$$
\hat{T}_{(u, \nu)} T \mathbb{U}_{0}=\left\{\left(w_{u}, w_{\nu}\right) \in T_{(u, \nu)} T \mathbb{U}_{0}: u \cdot \Omega w_{\nu}+w_{u} \cdot \Omega \nu=0\right\} .
$$

The inverse of $D \tilde{\chi}_{(u, \nu)}$ is undetermined. All the indeterminations discussed so far are fixed by choosing a smooth local inversion of $\pi$. Let us in fact consider a smooth local inversion of the map (8):

$$
\begin{aligned}
\pi^{-1} & : \mathcal{W} \longrightarrow \mathbb{U}_{0} \\
(x, y, z) & \longmapsto u=\pi^{-1}(x, y, z),
\end{aligned}
$$

with $\mathcal{W}$ open set. The choice of $\pi^{-1}$ uniquely fixes the inversion of equations (9), by providing:

$$
\begin{aligned}
u & =\pi^{-1}(x, y, z) \\
\nu & =\frac{1}{2} A^{T}(u)\left(v_{x}, v_{y}, v_{z}, 0\right),
\end{aligned}
$$

and, for tangent vectors $\left(V_{r}, V_{v}\right) \in \mathbb{R}^{8}$ with $V_{q}, V_{v_{q}}=0$, also the inversion of equations (44):

$$
\begin{aligned}
& w_{u}=J V_{r} \\
& w_{v}=\frac{1}{2} A^{T}(u) V_{v}-\frac{1}{\|u\|^{2}} A^{T}(u) A\left(w_{u}\right) \nu+\frac{2}{\|u\|^{2}} u \cdot w_{u} \nu
\end{aligned}
$$

where $J$ is the Jacobian matrix of the map $(x, y, z, q) \mapsto u=\pi^{-1}(x, y, z)$, computed at $(x, y, z, 0)$ $(u, \nu$ in the right hand side of (48) are obtained from (47)). 


\subsection{Variational equations of the restricted three-body problem}

Let us first consider the variational equations (17) of the regularized equations of motion (16). We notice that the vector field $F_{C}(\zeta)$ is invariant with respect to the transformation $R_{\alpha} \zeta=$ $\left(\mathcal{R}_{\alpha} u, \mathcal{R}_{\alpha} v\right)$, i.e. we have:

$$
F_{C}(\zeta)=R_{\alpha}^{T} F_{C}\left(R_{\alpha} \zeta\right) .
$$

Then, we consider the variational equations of the non-regularized equations of motion (1), obtained from the first order differential equations in the extended Cartesian space $\mathbb{R}^{8}$ :

$$
\left\{\begin{array}{l}
\dot{x}=v_{x}, \dot{y}=v_{y}, \dot{z}=v_{z}, \dot{q}=v_{q} \\
\dot{v_{x}}=2 v_{y}+x-(1-\mu) \frac{x+\mu}{r_{1}^{3}}-\mu \frac{x-1+\mu}{r_{2}^{3}} \\
\dot{v_{y}}=-2 v_{x}+y-(1-\mu) \frac{y}{r_{1}^{3}}-\mu \frac{y}{r_{2}^{3}} \\
\dot{v_{z}}=-(1-\mu) \frac{z}{r_{1}^{3}}-\mu \frac{z}{r_{2}^{3}} \\
\dot{v_{q}}=0
\end{array}\right.
$$

denoted in a compact form by:

$$
\dot{\xi}=\Xi(\xi)
$$

where, with a little abuse of notation, we denote $\xi=(r, v)=(x, y, z, q, \dot{x}, \dot{y}, \dot{z}, \dot{q})$; the extension to the $\left(q, v_{q}\right)$ variables is conveniently introduced to simplify the reference to the projection (44), of course we will only consider solutions with $q(t)=0, v_{q}(t)=0$. For any solution $\xi(t)$ of $(50)$ we obtain the variational equations:

$$
\dot{V}=\frac{\partial \Xi}{\partial \xi}(\xi(t)) V,
$$

where $V=\left(V_{r}, V_{v}\right)$. We prove the following:

Proposition 1. Let us consider a non constant solution $u(s)$ of equations (11) with initial conditions $\left(u(0), u^{\prime}(0)\right)$ satisfying condition (12), defined in some neighbourhood $\mathcal{S}$ of $s=0$ with $\pi(u(s)) \neq(-\mu, 0,0),(1-\mu, 0,0)$ for all $s \in \mathcal{S}$; denote by:

$$
t(s)=\int_{0}^{s}\|u(\sigma)\|^{2} d \sigma,
$$

and by $(x(t), y(t), z(t))$ the solution of (1) such that $(x(t(s)), y(t(s)), z(t(s)))=\pi(u(s))$ for all $s \in \mathcal{S}$. Then:

(i) there exist solutions $w(s)=\left(w_{u}(s), w_{\nu}(s)\right)$ of (17) defined by $\zeta(s)=\left(u(s), u^{\prime}(s)\right)$, with some $w(0) \in \hat{T} T \mathbb{U}$, such that by denoting with $V(t)=\left(V_{r}(t), V_{v}(t)\right)$ the functions of time defined by $V(t(s))=D \tilde{\chi}_{(u(s), \nu(s))}\left(w_{u}(s), w_{\nu}(s)\right)$ for all $s \in \mathcal{S}, V(t)$ is not a solution of (51).

(ii) let us consider a matrix $B(\zeta)$ such that, for any solution $\zeta(s)$ of (16) and for all the solutions $w(s)$ of the differential equation:

$$
w^{\prime}=B(\zeta(s)) w,
$$


the functions of time defined by $V(t(s))=D \tilde{\chi}_{(u(s), \nu(s))}\left(w_{u}(s), w_{\nu}(s)\right)$ are solutions of (51) with $\xi(t(s))=\tilde{\chi}(\zeta(s))$. Then, the matrix $B(\zeta)$ is singular at $u=0$.

From (i), we have that the variational equations of (1) are not conjugate by the KS transformation to the variational equations of the regularized equations of motion (11). This apparent contradiction is due to the fact that the Kustaanheimo Stiefel transformation, including the proper time transformation, is not simply a transformations of variables and therefore the usual conjugation of variational equations under smooth changes of variables does not apply. In particular, the chaos indicators constructed from the solutions of the variational equations, such as the Lyapunov exponents, the fast Lyapunov indicators, the finite time Lyapunov exponents, are different if they are defined from the solutions of (17) or from the solutions of (51). From (ii), we have that the equation conjugate to (51) is singular; i.e. the KS transformation regularizes the equations of motions, not the variational equations.

Proof of Proposition 1. For any solution $w(s)=\left(w_{u}(s), w_{\nu}(s)\right)$ of $(17)$ with $w(0) \in \hat{T} T \mathbb{U}$, since $u \cdot \Omega \nu$ is a first integral of equations (16), we have $w(s) \in \hat{T} T \mathbb{U}$ for all $s \in \mathcal{S}$. Let us consider the functions of time $V(t)=\left(V_{r}(t), V_{v}(t)\right)$ such that $V(t(s))=D \tilde{\chi}_{(u(s), \nu(s))}\left(w_{u}(s), w_{\nu}(s)\right)$ for all $s$. By computing the time derivative of $V_{r}(t)$, and using $w_{u}^{\prime}=w_{\nu}$, we obtain:

$$
\dot{V}_{r}=\frac{1}{\|u\|^{2}}\left(A(u) w_{\nu}+A(\nu) w_{u}+A\left(w_{\nu}\right) u+A\left(w_{u}\right) \nu\right)
$$

or equivalently:

$$
\dot{V}_{r}=V_{v}+\frac{4}{\|u\|^{4}} u \cdot w_{u} A(u) \nu
$$

Instead, the solutions $\tilde{V}(t)$ of (51) satisfy $\dot{\tilde{V}}_{r}=\tilde{V}_{v}$. Therefore, if we have:

$$
u(s) \cdot w_{u}(s) A(u(s)) \nu(s) \neq 0
$$

for some $s, V(t)$ does not solve (51). We remark that since the solution $u(s)$ is not constant, and $u(s) \neq 0$, we have $A(u(s)) \nu(s) \neq 0$ in an open subset $\tilde{\mathcal{S}}$ of $\mathcal{S}$. Therefore, if there exists $s \in \tilde{S}$ such that $u(s) \cdot w_{u}(s) \neq 0, V(t)$ does not solve (51). We now consider $w(0)$ such that $u(0) \cdot w_{u}(0) \neq 0$. Then, in a neighbourhood of $s=0$, we find $s \in \mathcal{S}$ where both $u(s) \cdot w_{u}(s) \neq 0$ and $A(u(s)) \nu(s) \neq 0$. This proves that solutions $w(s)$ of the variational equations (17) do not project on the solutions of the variational equations (51).

Then, let us suppose that the solutions of the differential equation:

$$
\left(w_{u}^{\prime}, w_{\nu}^{\prime}\right)=B(u(s), \nu(s))\left(w_{u}, w_{\nu}\right)
$$

project on the solution of the variational equations (51). Precisely, by defining $V(t)=\left(V_{r}(t), V_{v}(t)\right)$ such that $V(t(s))=D \tilde{\chi}_{(u(s), \nu(s))}\left(w_{u}(s), w_{\nu}(s)\right)$, then $V(t)$ satisfies (51). First, we notice that by 
computing the time derivative of $V_{r}(t)$ we obtain:

$$
\dot{V}_{r}=\frac{1}{\|u\|^{2}}\left(A(u) w_{u}^{\prime}+A(\nu) w_{u}+A\left(w_{u}^{\prime}\right) u+A\left(w_{u}\right) \nu\right) \text {. }
$$

From:

$$
\dot{V}_{r}=V_{v}=\frac{1}{\|u\|^{2}}\left(A(u) w_{\nu}+A\left(w_{u}\right) \nu+A\left(w_{\nu}\right) u+A(\nu) w_{u}-\frac{4 u \cdot w_{u}}{\|u\|^{2}} A(u) \nu\right)
$$

we obtain that, along all the solutions $w(s)$ of (53) we have

$$
A(u) w_{u}^{\prime}+A\left(w_{u}^{\prime}\right) u=A(u) w_{\nu}+A\left(w_{\nu}\right) u-\frac{4 u \cdot w_{u}}{\|u\|^{2}} A(u) \nu,
$$

and therefore the matrix $B(\zeta)$ satisfies:

$$
A(u)[B(\zeta) w]_{u}+A\left([B(\zeta) w]_{u}\right) u=A(u) w_{\nu}+A\left(w_{\nu}\right) u-\frac{4 u \cdot w_{u}}{\|u\|^{2}} A(u) \nu .
$$

If the matrix $B(\zeta)$ is regular at $u=0$, by taking the limit of $u$ tending to zero for constant $\nu, w$ of the previous equation we obtain:

$$
\lim _{u \rightarrow 0} \frac{4 u \cdot w_{u}}{\|u\|^{2}} A(u) \nu=0
$$

which is not satisfied for certain choices of $\nu, w_{u}$.

\subsection{Linearizations in the space of $\mathrm{KS}$ variables}

We need to establish some invariance properties of the Jacobian matrix $\frac{\partial F_{C}}{\partial \zeta}$ and of the solutions of the variational equations (17) with respect to the symmetry transformation (38). First, from the invariance relations (49), we obtain:

$$
\frac{\partial F_{C}}{\partial \zeta}(\zeta)=R_{\alpha}^{T} \frac{\partial F_{C}}{\partial \zeta}\left(R_{\alpha} \zeta\right) R_{\alpha}
$$

for any $\alpha$ and $\zeta$. As a consequence, by denoting with $\zeta(s), w(s)$ the solutions of the equations (16) and (17) with initial conditions $\zeta_{0}, w_{0}$, then $R_{\alpha} \zeta(s), R_{\alpha} w(s)$ are the solutions of (17) with initial conditions $R_{\alpha} \zeta_{0}, R_{\alpha} w_{0}$. This invariance will be used to define a chaos indicator invariant by the transformation $\zeta \mapsto R_{\alpha} \zeta$.

As a consequence of $(54)$, the eigenvalues of the Jacobian matrices $\frac{\partial F_{C}}{\partial \zeta}(\zeta)$ are invariant by the transformation $\zeta \mapsto R_{\alpha} \zeta$; i.e. they depend only on the projection $\mathcal{X}(\zeta)$. In particular, at all 
$\zeta=\left(u^{*}(\alpha), 0\right)$, corresponding to the representatives (40) of the Lagrangian points $L_{1}, L_{2}$, the matrix:

$$
F_{C, \alpha}=\frac{\partial F_{C}}{\partial \zeta}\left(u^{*}(\alpha), 0\right)
$$

has eigenvalues which are independent of $\alpha$ for all the values of $C$ : for $C=C_{i}\left(i=1,2\right.$ for $L_{1}$ or $L_{2}$ ) the matrices $F_{C_{i}, \alpha}$ have a pair of real eigenvalues $\pm \lambda^{0} \neq 0$, two pairs of purely complex eigenvalues $\pm i \sigma_{j}^{0}, j=1,2$, and an additional eigenvalue $\lambda=0$ of multiplicity 2 ; we also prove that ${ }^{7}$ for any fixed value of $\mu$ and $C=C_{i}-\varepsilon$, with suitably small $\varepsilon>0$, the matrix $F_{C, \alpha}$ has a pair of real eigenvalues $\pm \lambda^{\varepsilon}$, two pairs of purely complex eigenvalues $\pm i \sigma_{j}^{\varepsilon}, j=1,2$, and the real pair $\pm \sqrt{\varepsilon} / 2$.

In fact, by considering the linearization at $L_{1}$ (the case of $L_{2}$ is similar) for $C=C_{1}-\varepsilon$ with $\varepsilon>0$, since the eigenvalues are invariant with respect to the parameter $\alpha$, we only compute the eigenvalues of the matrix:

$$
F_{C, 0}=\left(\begin{array}{cc}
O & \mathcal{I}_{4} \\
\mathcal{A} & \mathcal{B}
\end{array}\right)
$$

where $O$ is a $4 \times 4$ square matrix with entries $O_{i j}=0$, for $i, j=1, \ldots, 4 ; \mathcal{I}_{4}$ is the $4 \times 4$ identity matrix, the matrices $\mathcal{A}, \mathcal{B}$ are defined by:

$$
\mathcal{A}=\left(\begin{array}{llll}
a & 0 & 0 & 0 \\
0 & b & 0 & 0 \\
0 & 0 & c & 0 \\
0 & 0 & 0 & d
\end{array}\right) \quad, \quad \mathcal{B}=\left(\begin{array}{cccc}
0 & 2 \gamma_{1} & 0 & 0 \\
-2 \gamma_{1} & 0 & 0 & 0 \\
0 & 0 & 0 & 0 \\
0 & 0 & 0 & 0
\end{array}\right)
$$

where:

$$
\begin{aligned}
a & =\frac{\varepsilon}{4}-\gamma_{1}^{2} \frac{3+\gamma_{1}^{2}-2 \gamma_{1}^{3}+\gamma_{1}^{4}}{\gamma} \\
b & =\frac{\varepsilon}{4}-\gamma_{1}^{2} \frac{-9+6 \gamma_{1}-5 \gamma_{1}^{2}-2 \gamma_{1}^{3}+\gamma_{1}^{4}}{\gamma} \\
c & =\frac{\varepsilon}{4} \\
d & =\frac{\varepsilon}{4}-2 \gamma_{1}^{2} \frac{2-\gamma_{1}+\gamma_{1}^{2}}{\gamma} \\
\gamma & =1-2 \gamma_{1}+\gamma_{1}^{2}+2 \gamma_{1}^{3}-\gamma_{1}^{4} .
\end{aligned}
$$

The characteristic equation determining the eigenvalues $\lambda$ of (56):

$$
\operatorname{det}\left(\mathcal{A}+\lambda \mathcal{B}-\lambda^{2} \mathcal{I}_{4}\right)=0
$$

\footnotetext{
${ }^{7}$ Since we will consider the dynamics in small neighbourhoods of $L_{1}$ (or $L_{2}$ ) for values of the Jacobi constant smaller than $C_{1}$ (or $C_{2}$ ), we will consider expansions of $\frac{\partial F_{C}}{\partial \zeta}(\zeta)$ at $\zeta=\left(u^{*}(\alpha), 0\right)$ also for $C<C_{i}$.
} 
can be represented in the form:

$$
\frac{1}{\gamma^{2}}\left(\frac{\varepsilon}{4}-\lambda^{2}\right)\left(-\frac{2 \gamma_{1}^{2}\left(2-\gamma_{1}+\gamma_{1}^{2}\right)}{\gamma}+\frac{\varepsilon}{4}-\lambda^{2}\right)\left(R_{1}+R_{2} \lambda^{2}+R_{3} \lambda^{4}\right)=0 .
$$

where $R_{1}, R_{2}$ are coefficients depending only on $\gamma_{1}, \varepsilon$ and $R_{3}$ dependent only of $\gamma_{1}$. Therefore, four eigenvalues are:

$$
\begin{aligned}
& \lambda_{1,2}= \pm \sqrt{\varepsilon} \\
& \lambda_{3,4}= \pm i \sqrt{\frac{2 \gamma_{1}^{2}\left(2-\gamma_{1}+\gamma_{1}^{2}\right)}{\gamma}-\frac{\varepsilon}{4}}
\end{aligned}
$$

while the other four eigenvalues are:

$$
\lambda_{5,6,7,8}= \pm \sqrt{\frac{-R_{2} \pm \sqrt{R_{2}^{2}-4 R_{1} R_{3}}}{2 R_{3}}} .
$$

Since for small $\varepsilon$ we have $R_{2}<0, R_{3}>0$, and $R_{1} R_{3}<0$, two of the eigenvalues (61) are opposite real numbers, the other two are opposite purely imaginary numbers.

Remark. The explicit computations of Section 6 will be done for values of $\mu$ corresponding the the Sun-Jupiter system and a value of the Jacobi constant $C=3.03685 \ldots$; correspondingly we have:

$$
\frac{2 \gamma_{1}^{2}\left(2-\gamma_{1}+\gamma_{1}^{2}\right)}{\gamma}-\frac{\varepsilon}{4}>0
$$

as well as $R_{2}<0, R_{3}>0$, and $R_{1} R_{3}<0$, so that the above analysis is applicable.

\section{Conclusions}

The global computations of the manifolds asymptotic to the center manifolds originating at the Lagrangian points $L_{1}, L_{2}$ is a problem at the frontier of the dynamical systems science; in fact, in addition to usual numerical difficulties induced by hyperbolic dynamics, the gravitational singularities determine a non trivial geometry of the phase-space and a source of numerical limitations. Geometric regularizations, such as the Kustaanheimo-Stiefel regularizations, provide excellent solutions to the numerical issues, but increase the dimension of the phase space with subtle consequences. In this paper, we abandon the traditional way of computing the asymptotic manifolds for a strategy based on the use of chaos indicators. We had to formulate from scratch the variational theory related to the Kustaanheimo-Stiefel regularizations, especially to study the relationship with the orbits in the physical phase space. We found natural to define the chaos indicators in a geometric framework. The applications of these ideas to the Sun-Jupiter case have provided new insights about the structures of the asymptotic manifolds as well as of 
the dynamics in the vertical section $\Sigma_{V}$. We plan in the future to consider further extension of this theory, by including perturbations of the spatial three-body problem, such as elliptic problems or perturbations from an additional massive body.

\section{Acknowledgments}

We are deeply grateful to Claude Froeschlé, who suggested us in 2008 to define chaos indicators using the KS transformation. We needed eight years to fill the gaps between some promising numerical results produced at the time and the results here reported, but without Claude's intuition the present paper would not exist. M. Guzzo gas been supported by the project CPDA149421/14 of the University of Padova "New Asymptotic Aspects of Hamiltonian Perturbation Theory". Part of the numerical computations have been done on the "Mesocentre SIGAMM" machine, hosted by the Observatoire de la Côte d'Azur. The three-dimensional graphics of figures 3,5,9,10 have been produced using the software Meshlab, by Visual Computing Lab-ISTI-CNR, http://meshlab.sourceforge.net/, (a tool developed with the support of the 3D-CoForm project).

\section{References}

[1] Bettis D. G. and Szebehely V., Treatment of Close Approaches in the Numerical Integration of the Gravitational Problem of N-Bodies (Papers appear in the Proceedings of IAU Colloquium No. 10 Gravitational N-Body Problem (ed. by Myron Lecar), R. Reidel Publ. Co. , Dordrecht-Holland.), Ap \& SS, 14, 133, 1971.

[2] Conley C., Low Energy Transit Orbits in the Restricted Three-Body Problems. SIAM J. Appl. Math., 16(4), 732746, 1967.

[3] Conley C., Easton R., Isolated invariant sets and isolating blocks, Transactions of the American Mathematical Society 158, 35-61, 1971.

[4] Anderson R.L., Easton R.W. and Lo M.W., Isolating blocks as computational tools in the circular restricted three-body problem, Physica D, 343, 38-50, 2017.

[5] Froeschlé C., Numerical Studies of Dynamical Systems with Three Degress of Freedom, Astron. \& Astrophys., vol. 4, 115-128, 1970.

[6] C. Froeschlé, M. Guzzo and E. Lega, Graphical Evolution of the Arnold Web: From Order to Chaos. Science, 289, n. 5487: 2108-2110, 2000.

[7] Froeschlé C., Lega E. and Gonczi R., Fast Lyapunov indicators. Application to asteroidal motion, Celest. Mech. and Dynam. Astron., 67, pp. 41-62, 1997. 
[8] Gawlik E.S., Marsden J.E., Du Toit P., Campagnola S., Lagrangian coherent structures in the planar elliptic restricted three body problem, Celestial Mech. Dynam. Astronom., 103, $227249,2009$.

[9] Gomez G., Koon W.S., Lo M.W., Marsden J.E., Masdemont J. and Ross S.D., Connecting orbits and invariant manifolds in the spatial restricted three-body problem, Nonlinearity, 17, 15711606, 2004.

[10] Guzzo M., Chaos and diffusion in dynamical systems through stable-unstable manifolds, in "Space Manifolds Dynamics: Novel Spaceways for Science and Exploration", proceedings of the conference: "Novel spaceways for scientific and exploration missions, a dynamical systems approach to affordable and sustainable space applications" held in Fucino Space Centre (Avezzano) 15-17 October 2007. Editors: Perozzi and Ferraz Mello. Springer. 2010.

[11] Guzzo M., Lega E. and Froeschlé C., A numerical study of the topology of normally hyperbolic invariant manifolds supporting Arnold diffusion in quasi-integrable systems. Physica D,182, 1797-1807, 2009.

[12] Guzzo M., Lega E., On the identification of multiple close-encounters in the planar circular restricted three body problem." Monthly Notices of the Royal Astronomical Society, 428, 2688-2694, 2013.

[13] Guzzo M., Lega E., Evolution of the tangent vectors and localization of the stable and unstable manifolds of hyperbolic orbits by Fast Laypunov Indicators, SIAM J. APPL. MATH., Vol. 74, No. 4, pp. 10581086, 2014.

[14] Guzzo M., Lega E., A study of the past dynamics of comet 67P/Churyumov-Gerasimenko with Fast Lyapunov Indicators, Astronomy \& Astrophysics, vol. 579, A79, 1-7, 2015.

[15] Gómez G., Jorba À., Masdemont J., Simó C., Dynamics and Mission Design Near Libration Point Orbits, Vol. 3: Advanced Methods for Collinear Points, World Scientific, Singapore, 2000 .

[16] Jorba A., Masdemont J., Dynamics in the center manifold of the restricted three-body problem, Physica D 132, 189213, 1999.

[17] Koon W.S., Lo M.W., Marsden J.E. and Ross S.D., Dynamical Systems, the three body problem and space mission design. Marsden Books. ISBN 978-0-615-24095-4, 2008.

[18] Kustaanheimo P., Spinor regularisation of the Kepler motion, An- nales Universitatis Turkuensis A 73, 1-7. Also Publications of the As- tronomical Observatory Helsinki 102, 1964. 
[19] Kustaanheimo P. and Stiefel E.L., Perturbation theory of Kepler motion based on spinor regularization, Journal f ur die Reine und Angewandte Mathematik 218, 204-219, 1965.

[20] Haller, G., Distinguished material surfaces and coherent structures in three-dimensional fluid flows, Physica D 149, 248277, 2000.

[21] Haller, G., Lagrangian coherent structures from approximate velocity data. Physics of Fluids, 14 (6), 18511861, 2002.

[22] Lega E., Guzzo M. and Froeschlé C., A numerical study of the hyperbolic manifolds in a priori unstable systems. A comparison with Melnikov approximations, Cel. Mech. and Dyn. Astron., 107, pp. 115-127, 2010.

[23] Lega E., Guzzo M. and Froeschlé C., Detection of close encounters and resonances in threebody problems through Levi-Civita regularization, Monthly Notices of the Royal Astronomical Society, vol. 418, 107-113, 2011.

[24] Lega E. and Guzzo M., Three-dimensional representations of the tube manifolds of the planar restricted three-body problem, Physica D, vol. 325, 41-52, 2016.

[25] Lega E. and Guzzo M., Theory and Applications of the Fast Lyapunov Indicator (FLI) Method, in: Lecture Notes in Physics 915, Chaos Detection and Predictability, pp.35-54, 2016.

[26] Lekien, F., Shadden, S.C., and Marsden, J.E., Lagrangian coherent structures in ndimensional systems. Journal of Mathematical Physics, 48, 065404, 2007.

[27] Levi-Civita T., Sur la régularisation qualitative du probléme restreint des trois corps, Acta Math., vol. 30, 305-327, 1906.

[28] Moeckel R., Isolating Blocks Near the Collinear Relative Equilibria of the Three-Body Problem, Transactions of the American Mathematical Society 356, 4395-4425, 2004.

[29] Roy A. and Ovenden M.W., On the occurrence of commensurable mean motions in the Solar System II, The mirror theorem, Mon. Not. Roy. Astr. Soc. 115, pag. 296, 1955.

[30] Shadden S.C., Lekien F. and Marsden J.E., Definition and properties of Lagrangian coherent structures from finite-time Lyapunov exponents in two-dimensional aperiodic flows, Physica D 212, 271304, 2005.

[31] Sijbrand J., Properties of Center Manifolds, Transactions of the American Mathematical Society, vol. 289, n. 2, pp. 431-469, 1985. 
[32] Simó C., Dynamical systems methods for space missions on a vicinity of collinear libration points, in Simó, C., editor, Hamiltonian Systems with Three or More Degrees of Freedom (S'Agaró, 1995), volume 533 of NATO Adv. Sci. Inst. Ser. C Math. Phys. Sci., pp. 223-241, Dordrecht. Kluwer Acad. Publ., 1999.

[33] Szebehely V., Theory of orbits. Academic Press, New York, 1967.

[34] Sundman K. F., Acta Math., 36, 105, 1912.

[35] Waldvogel J., Fundamentals of Regularization in Celestial Mechanics and Linear Perturbation Theories, in Extra-Solar Planets : The Detection, Formation, Evolution and Dynamics of Planetary Systems, B. Steves, M. Hendry, A.C. Cameron editors, CRC Press, Taylor and Francis group, 2001.

[36] Tang X.Z., Boozer A.H., Finite time Lyapunov exponent and advectiondiffusion equation, Physica D 95 (1996) 283305.

[37] Villac B.F., Using FLI maps for preliminary spacecraft trajectory design in multi-body environments, Cel. Mech. and Dyn. Astron., 102, pp. 29-48, 2008. 